FOLIA

Amazónica

Revista del Instituto de Investigaciones

de la Amazonía Peruana

\title{
DIVERSIDAD DE ANFIBIOS Y REPTILES EN EL INTERFLUVIO PUTUMAYO-NAPO-AMAZONAS, AL NORTE DE LA AMAZONÍA PERUANA
}

\author{
Carlo Jaminton TAPIA DEL ÁGUILA ${ }^{1, *}$, Jhon Jairo LÓPEZ-ROJAS ${ }^{2}$, \\ Pedro E. PÉREZ-PEÑA ${ }^{3}$
}

1 Universidad Nacional de la Amazonía Peruana (UNAP), Facultad de Ciencias Biológicas. Iquitos, Perú.

2 Estación Experimental Agraria El Porvenir. Dirección de Desarrollo Tecnológico Agrario, Instituto Nacional de Innovación Agraria (INIA), Jr. Martinez de Compagñon 1035, Tarapoto, San Martín 22200, Perú.

3 Instituto de Investigaciones de la Amazonía Peruana (IIAP).

* Correo electrónico: cayotapia@gmail.com

\section{RESUMEN}

El conocimiento de la biodiversidad de anfibios y reptiles es fundamental para proponer la conservación de estos taxa. En este trabajo se realizaron registros por encuentros visuales y recopilación bibliográfica para conocer la diversidad de anfibios y reptiles por tipos de hábitats en el interfluvio Putumayo-NapoAmazonas. Nuestros resultados muestran la existencia de 140 especies de anfibios y 108 reptiles. El bosque de colina tuvo mayor riqueza de especies (58 especies de anfibios y 40 de reptiles). Las familias con mayor riqueza fueron Hylidae (38 especies) y Colubridae (39 especies). El análisis de similitud de hábitats en anfibios mostró dos grupos: de los bosques de terraza alta, terraza media y bosque de colinas y del bosque de terraza baja, aguajal y varillal pantanoso. En los reptiles también se formaron dos grupos: los de aguajal, bosque de colinas y terraza alta, y de los bosques de terraza baja, terraza media y varillal pantanoso. En este interfluvio, la herpetofauna está amenazada por la deforestación, la agricultura, la cacería de caimanes y recolección de huevos de tortugas. Si estas presiones antrópicas se intensifican podrían causar drásticas reducciones poblacionales.

PALABRAS CLAVE: Abundancia, amenazas, herpetofauna, riqueza, similitud. 


\title{
AMPHIBIAN AND REPTILE DIVERSITY IN THE NAPO-PUTUMAYO-AMAZONAS INTERFLUVIUM, NORTHERN PERUVIAN AMAZON
}

\begin{abstract}
Knowledge of the biodiversity of amphibians and reptiles is essential in developing conservation for these taxa. We collected data from visual encounters and bibliographic review to determine the diversity of amphibians and reptiles in different habitats in the Putumayo-Napo-Amazonas interfluvium. This interfluvium presents 140 species of amphibians and 108 reptiles. The hill forest had the highest species richness, with 58 species of amphibians and 40 species of reptiles. The richest families were Hylidae and Colubridae. The Hilids presented a greater number of species in flooded ecosystems, while the Craugastoridae and Bufonidae families had greater richness in non-flooded ones. The Colubridae family was present in both ecosystems, although with a greater presence in flooded habitats. A community similarity analysis showed two groups: one formed by the amphibians of the high terrace, middle terrace and hill forest forests, and a second group from the low terrace, palm swamp, and peatland pole forest. In the reptiles, two groups were also formed: one by the reptiles of the palm swamp, hill forest and high terrace, and the second group made up of the reptiles of the low terrace forest, middle terrace and peatland pole forest. This diversity is threatened by deforestation, agriculture, caiman hunting and turtle egg collection, if intensified, could cause drastic population declines in these species.
\end{abstract}

KEYWORDS: Abundance, herpetofauna, richness, similarity, threats. 


\section{INTRODUCCIÓN}

El interfluvio Putumayo-Napo-Amazonas se encuentra entre las ecorregiones prioritarias de conservación en el neotrópico (Loyola et al., 2009) formando parte de las ecorregiones del Napo y Solimões-Japurá del bioma amazónico (Dinerstein et al., 2017). Aproximadamente el $20 \%$ de su extensión se encuentra en protección (parques, áreas de conservación y reserva comunal) desde los últimos 20 años (Maretti et al., 2014), existiendo actividades antrópicas de impacto negativo sobre las especies de flora y fauna.

Durante las últimas décadas, diversos estudios de biodiversidad en los ecosistemas de la Amazonía norte peruana han sido de vital importancia para promover estrategias de conservación (Lescure \& Gasc, 1986; Pitman et al., 2004; Alverson et al., 2008; Gilmore et al., 2010; Pitman et al., 2011; Knell, 2012; Pitman et al., 2013; Pitman et al., 2016; Pérez-Peña et al., 2019a; Pérez-Peña et al., 2019b; Pérez-Peña et al., en prensa). Al mismo tiempo, permitió obtener un panorama situacional de las especies indicadoras en determinados ecosistemas, siendo los anfibios y reptiles, grupos sensibles a modificaciones antrópicas de sus hábitats (da Cunha et al., 2015; Gallmetzer \& Schulze, 2015).

Actualmente en el Perú, se registra un total de 664 especies de anfibios y 506 de reptiles, cifra que se ha elevado enormemente en los últimos años (Aguilar et al., 2010; Frost, 2020; Uetz et al., 2020) como resultado de la descripción de nuevas especies, incremento de colecciones e investigaciones científicas que contribuyen en cubrir vacíos de información (Ossa et al., 2012; OTCA, 2018).

De esta manera, con la descripción de nuevas especies, revalidación en sinonimias o cambios taxonómicos a nivel de género y ampliación de distribución, tanto en anfibios (Pramuk, 2006; Elmer \& Cannatella, 2008; Lehr et al., 2010; Moravec et al., 2010; Wiens et al., 2010; Brown et al., 2011; López-Rojas \& Cisneros-Heredia, 2012; Caminer \& Ron, 2014; de Sá et al., 2014; Ortega-Andrade \& Venegas, 2014; Rojas et al., 2015; Duellman et al., 2016; Dubois, 2017; Rojas et al., 2018) como en reptiles (Miralles et al., 2005, 2006, 2009; Bergmann \& Russell, 2007; Carrasco et al., 2012; Grazziotin et al., 2012; Hedges \& Conn, 2012; Köhler et al., 2012; Olson \& David, 2014; Goicoechea et al., 2016) del noreste del Departamento de Loreto, Perú. Se ha visto necesario la verificación y actualización de la lista de especies de herpetofauna en diversos inventarios realizados a lo largo del interfluvio del Putumayo-Napo-Amazonas. Entre algunas localidades se encuentran: Yubineto-Ampiyacu (Lescure \& Gasc, 1986), Ampiyacu-Apayacu (Rodríguez \& Knell, 2004), Cuyabeno-Güeppí (Yáñez-Muñoz \& Venegas, 2008), Maijuna (von May \& Venegas, 2010), Yaguas-Cotuhé (von May \& Mueses-Cisneros, 2011), Lagartococha (Knell, 2012), Ere-Campuya-Algodón (Venegas \& Gagliardi-Urrutia, 2013), Medio PutumayoAlgodón (Chávez \& Mueses-Cisneros, 2016), Alto Putumayo (Pérez-Peña et al., 2019c), NapoCuraray (Tapia-del Águila et al., 2019), Bajo Putumayo (Tapia-del Águila \& Pérez-Peña, en prensa).

El presente estudio tiene como finalidad conocer la diversidad de anfibios y reptiles en el interfluvio Putumayo-Napo-Amazonas y en sus hábitats, actualizar la lista de especies y cambios taxonómicos, su estado de conservación e identificar las posibles amenazas que pueden afectarla. Esta información podrá ser utilizada en futuras investigaciones para implementar planes de conservación de la herpetofauna y de otros componentes que forman parte de la diversidad biológica. 


\section{MATERIAL Y MÉTODOS}

\section{ÁREA DE ESTUDIO}

Se utilizó datos publicados de estudios realizados en el interfluvio Putumayo-Napo-Amazonas (Figura 1). Las zonas evaluadas exhiben una temperatura anual mínima de $20,9{ }^{\circ} \mathrm{C}$ y máxima de $31,7{ }^{\circ} \mathrm{C}, 88 \%$ de humedad relativa promedio y una precipitación mensual de 200 a $300 \mathrm{~mm}$
(Climate-Data, 2018). La temporada de creciente se inicia en marzo, llegando a su pico más alto entre mayo y julio, la vaciante comienza en agosto y culmina en febrero, llegando a su nivel más bajo en noviembre.

\section{MÉTODOS}

El estudio tuvo dos tipos de métodos: registro por encuentros visuales y revisión bibliográfica.

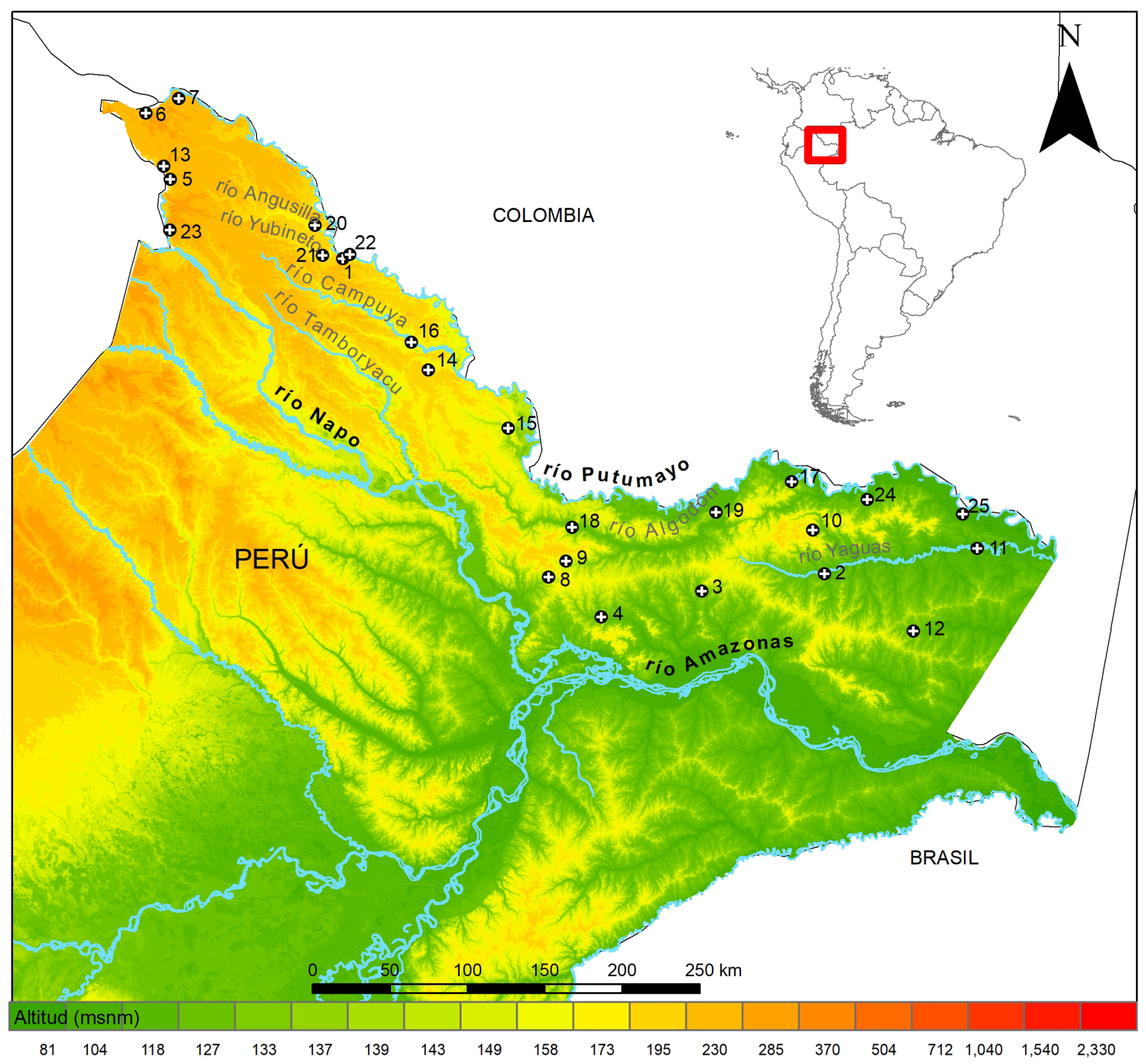

Figura 1: Mapa de ubicación de los estudios herpetológicos realizados en el interfluvio Putumayo-Napo-Amazonas. Los números representan las localidades de muestreo: 1. Yubineto, 2. Yaguas,

3. Maronal, 4. Apayacu, 5. Redondococha, 6. Güeppí, 7. Aguas Negras, 8. Curupa, 9. Piedras, 10. Choro,

11. Cachimbo, 12. Alto Cotuhé, 13. Lagartococha, 14. Ere-Algodón, 15. Bajo Ere, 16. Medio Campuya,

17. Quebrada Bufeo, 18. Medio Algodón, 19. Bajo Algodón, 20. Mashunta, 21. Santa Rita, 22. Nuevo Jerusalén, 23. Río Aguarico, 24. Remanso, 25. Tres Esquinas. 


\section{REGISTRO POR ENCUENTROS VISUALES}

Se usó este método para registrar la mayor cantidad de especies (Doan, 2003) y estimar la riqueza por hábitat. La búsqueda se realizó en transectos de $200 \mathrm{~m}$ previamente establecidos en seis hábitats de seis localidades (Mashunta, Santa Rita, Nueva Jerusalen, Tres Esquinas, Remanso y Aguarico). El esfuerzo realizado en cada hábitat fue 7,4 km en colina baja, 5,6 km en terraza alta, $8,8 \mathrm{~km}$ en terraza media, $3,2 \mathrm{~km}$ en terraza baja, $3,6 \mathrm{~km}$ en aguajal y $2,0 \mathrm{~km}$ en varillal pantanoso. La búsqueda consistía en remover la hojarasca, ramas o troncos caídos, revisando plántulas, arbustos, raíces de árboles, troncos delgados y zonas potenciales para reproducción (Crump \& Scott, 2001). En cada registro se anotaron el nombre de la especie, número de individuos, microhábitat y hábitat. Los muestreos diurnos se realizaron desde las 8:00 hasta las 12:00 horas y los nocturnos desde las 19:00 hasta las 23:00 horas, pudiendo variar en días de lluvias.

\section{RECOPILACIÓN BIBLIOGRÁFICA}

Se realizó una recopilación bibliográfica de las especies registradas en el interfluvio PutumayoNapo-Amazonas. Esta base de datos fue sometida a diferentes procesos de filtrados y revisiones taxonómicas, teniendo en cuenta la distribución de cada especie y cambios taxonómicos que tuvieron desde su publicación hasta la actualidad y considerando a los registros más confiables. La nomenclatura para anfibios sigue a Frost (2020) y para reptiles a Uetz et al. (2020).

Se utilizó la información publicada proveniente de inventarios herpetológicos de los ríos Yaguas, Cotuhé, Apayacu, Algodón, Ere, Campuya, Yubineto, Lagartococha, Redondococha, Aguas Negras, Güeppí y Aguarico (Lescure \& Gasc, 1986; Rodríguez \& Knell, 2004; Yáñez-Muñoz
\& Venegas, 2008; von May \& Venegas, 2010; von May \& Mueses-Cisneros, 2011; Knell, 2012; Venegas \& Gagliardi-Urrutia, 2013; Chávez \& Mueses-Cisneros, 2016; Pérez-Peña et al., 2019c; Tapia-del Águila et al., 2019; Tapia-del Águila \& Pérez-Peña, en prensa) (Tabla 1).

\section{ANÁLISIS DE DATOS}

La riqueza global se estimó a través del conteo de especies registradas en las diferentes publicaciones de la zona y mediante registros por encuentros visuales. Mientras que la riqueza observada por hábitats fue obtenida de los registros por encuentros visuales en las seis localidades: Mashunta, Santa Rita, Nueva Jerusalén, Tres Esquinas, Remanso y Aguarico; y se aplicó un índice de rarefacción por número de individuos para comparar la riqueza observada por tratarse de muestras desiguales. Asimismo, se utilizó el estimador no paramétrico de Chao 1 para estimar la riqueza esperada en cada uno de estos hábitats. Del mismo modo, la similitud de especies fue analizada mediante el índice de similitud de Sorensen. Todos los datos fueron analizados y graficados en el software RStudio (RStudio Team, 2019).

Se identificaron, analizaron y compararon seis tipos de hábitats donde los anfibios y reptiles estuvieron presentes: bosque de aguajal, bosque de colina baja, bosque de terraza alta, media y baja, y bosque de varillal pantanoso, siguiendo la clasificación de MINAM (2015) y Zarate et al. (2019). La identificación de las categorías de amenaza de anfibios y reptiles fue realizada mediante el uso del listado de especies amenazadas a nivel nacional del DS-004-2014MINAGRI (MINAGRI, 2014), y las categorías internacionales de la CITES (2020) y la lista roja de la IUCN (2020). 
Tabla 1: Lista de fuentes de información, lugares de muestreo y coordenadas de ubicación. Los números $\left(\mathrm{N}^{\circ}\right)$ están de acuerdo con el mapa (Figura 1).

\begin{tabular}{|c|c|c|c|c|}
\hline Autor & $\mathbf{N}^{\circ}$ & Zonas de muestreo & Latitude & Longitude \\
\hline 1. Lescure \& Gasc (1986) & 1 & Yubineto & $-1,033,333$ & $-74,216,667$ \\
\hline \multirow{3}{*}{ 2. Rodríguez \& Knell (2004) } & 2 & Yaguas & $-2,864,861$ & $-71,415,028$ \\
\hline & 3 & Maronal & -2.965639 & $-72,127,861$ \\
\hline & 4 & Apayacu & $-3,116,667$ & $-72,712,500$ \\
\hline \multirow{3}{*}{ 3. Yáñez-Muñoz \& Venegas (2008) } & 5 & Redondococha & $-0,571306$ & $-75,219,222$ \\
\hline & 6 & Güeppí & $-0,184694$ & $-75,358,972$ \\
\hline & 7 & Aguas Negras & $-0,100445$ & $-75,167,972$ \\
\hline \multirow{2}{*}{ 4. von May \& Venegas (2010) } & 8 & Curupa & $-2,885,028$ & $-73,018,667$ \\
\hline & 9 & Piedras & $-2,792,750$ & $-72,917,472$ \\
\hline \multirow{4}{*}{ 5. von May \& Mueses-Cisneros (2011) } & 10 & Choro & $-2,610,611$ & $-71,485,750$ \\
\hline & 2 & Yaguas & $-2,864,861$ & $-71,415,028$ \\
\hline & 11 & Cachimbo & $-2,718,306$ & $-70,529,194$ \\
\hline & 12 & Alto Cotuhé & $-3,198,778$ & $-70,899,028$ \\
\hline 6. Knell (2012) & 13 & Lagartococha & $-0,493526$ & $-75,257,066$ \\
\hline \multirow{3}{*}{ 7. Venegas \& Gagliardi-Urrutia (2013) } & 14 & Ere- Algodón & $-1,679,028$ & $-73,719,694$ \\
\hline & 15 & Bajo Ere & $-2,018,722$ & $-73,253,722$ \\
\hline & 16 & Medio Campuya & $-1,517,611$ & $-73,816,167$ \\
\hline \multirow{3}{*}{ 8. Chávez \& Mueses-Cisneros (2016) } & 17 & Quebrada Bufeo & $-2,330,611$ & $-71,607,528$ \\
\hline & 18 & Medio Algodón & $-2,595,056$ & $-72,884,056$ \\
\hline & 19 & Bajo Algodón & $-2,506,103$ & $-72,047,981$ \\
\hline \multirow{3}{*}{ 9. Pérez-Peña et al. (2019c) } & 20 & Mashunta & $-0,837507$ & $-74,376,524$ \\
\hline & 21 & Santa Rita & $-1,013,419$ & $-74,332,619$ \\
\hline & 22 & Nuevo Jerusalén & $-1,009,051$ & $-74,175,631$ \\
\hline 10. Tapia-del Águila et al. (2019) & 23 & Río Aguarico & $-0,866133$ & $-75,220,675$ \\
\hline \multirow{2}{*}{ 11. Tapia-del Águila \& Pérez-Peña (En prensa) } & 24 & Remanso & $-2,432,964$ & $-71,167,500$ \\
\hline & 25 & Tres Esquinas & $-2,518,071$ & $-70,612,703$ \\
\hline
\end{tabular}




\section{RESULTADOS}

\section{RIQUEZA DE ESPECIES}

El interfluvio Putumayo-Napo-Amazonas alberga 248 especies delas cuales 140 sonanfibiosy 108 son reptiles (Figura 2). Los anfibios están distribuidos en tres órdenes y 14 familias, siendo Hylidae (38 especies) y Craugastoridae (32 especies), las familias con mayor riqueza. Los reptiles se distribuyeron en tres órdenes y 21 familias, siendo Colubridae (39 especies) la familia con mayor riqueza, otras familias con mayor número de especies fueron Elapidae y Gymnophtalmidae con ocho especies cada una (Tabla 2).

El análisis de riqueza por hábitats indicó que el bosque de colina baja tuvo mayor número de especies de anfibios. En este bosque se registraron 58 especies y se espera que existan 69 especies, estos cálculos demuestran un porcentaje de efectividad de 84\% (Figura 3). De la misma manera en reptiles, el bosque de colina baja fue el hábitat de mayor riqueza, con 40 especies registradas (Tabla 3). Sin embargo, de acuerdo al estimador no paramétrico utilizado, la riqueza esperada fue de 105 especies, lo cual representa menos del $38 \%$ de efectividad de muestreo (Figura 3). El bosque de aguajal y el de terraza media para los anfibios y el bosque de terraza alta para reptiles, presentaron porcentajes de efectividad por encima del $70 \%$, a diferencia de los demás hábitats que presentaron porcentajes menores (Figura 3).

La riqueza herpetológica por familia y hábitats mostró una gran riqueza de las familias Hylidae en anfibios y Colubridae en reptiles. La familia Hylidae fue más dominante en ambos ecosistemas, mientras que en los ecosistemas de tierra firme se notó también una importante presencia de las familias Craugastoridae y Bufonidae. En el caso de los reptiles, la familia Colubridae tuvo mayor presencia en los ecosistemas de tierra firme y muy poco en los bosques inundables (Figura 4).
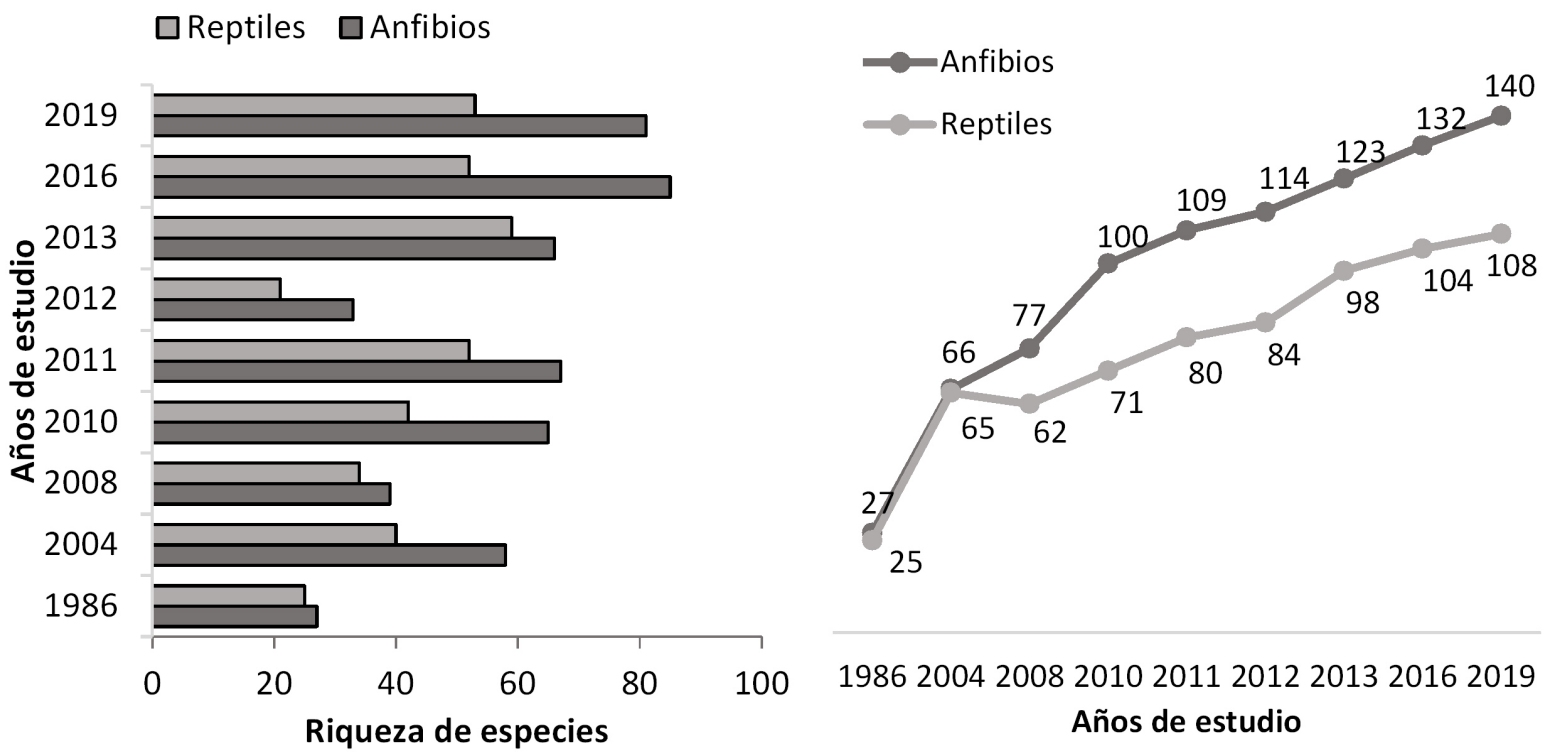

Figura 2: Número de especies de anfibios y reptiles registrados desde 1986 al 2019 (izquierda) y su curva de acumulación en el interfluvio Putumayo-Napo-Amazonas (derecha). Los registros se obtuvieron de Lescure \& Gasc (1986); Rodríguez \& Knell (2004); Yáñez-Muñoz \& Venegas (2008); von May \& Venegas (2010); von May \& Mueses-Cisneros (2011); Knell (2012); Venegas \& Gagliardi-Urrutia (2013); Chávez \& Mueses-Cisneros (2016); Pérez-Peña et al. (2019c); Tapia-del Águila et al. (2019); Tapia-del Águila \& Pérez-Peña (en prensa). 

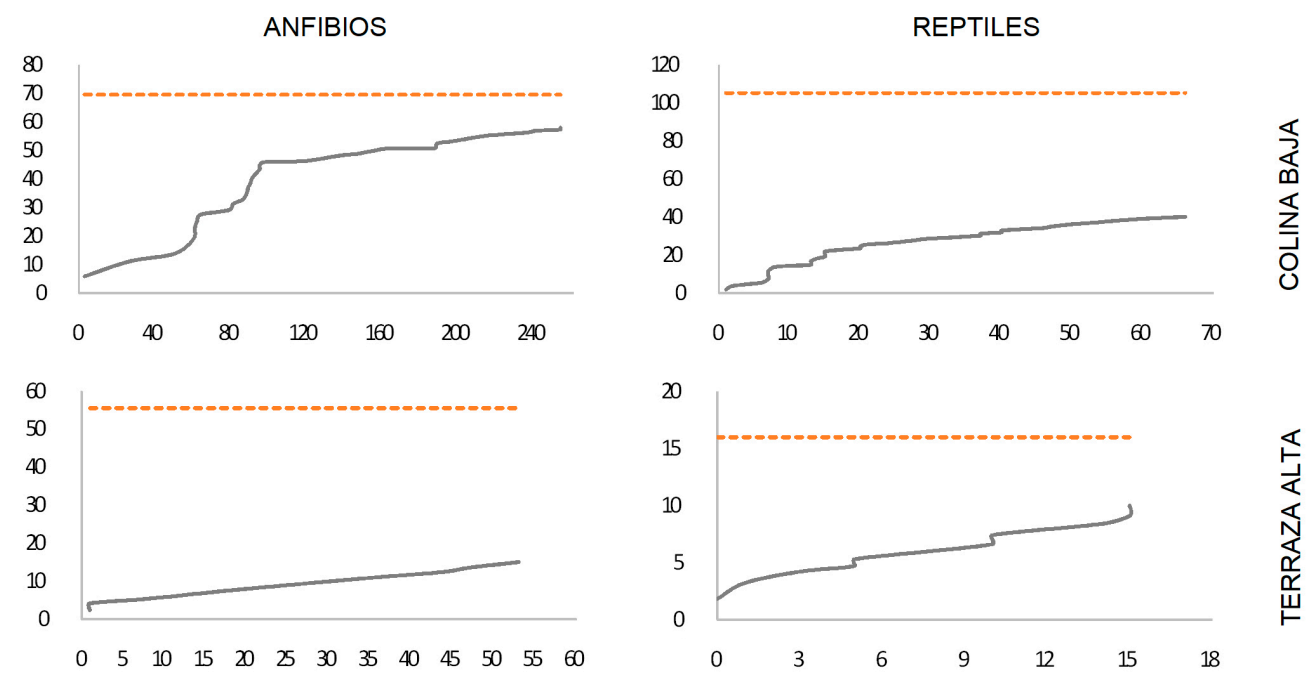

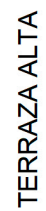
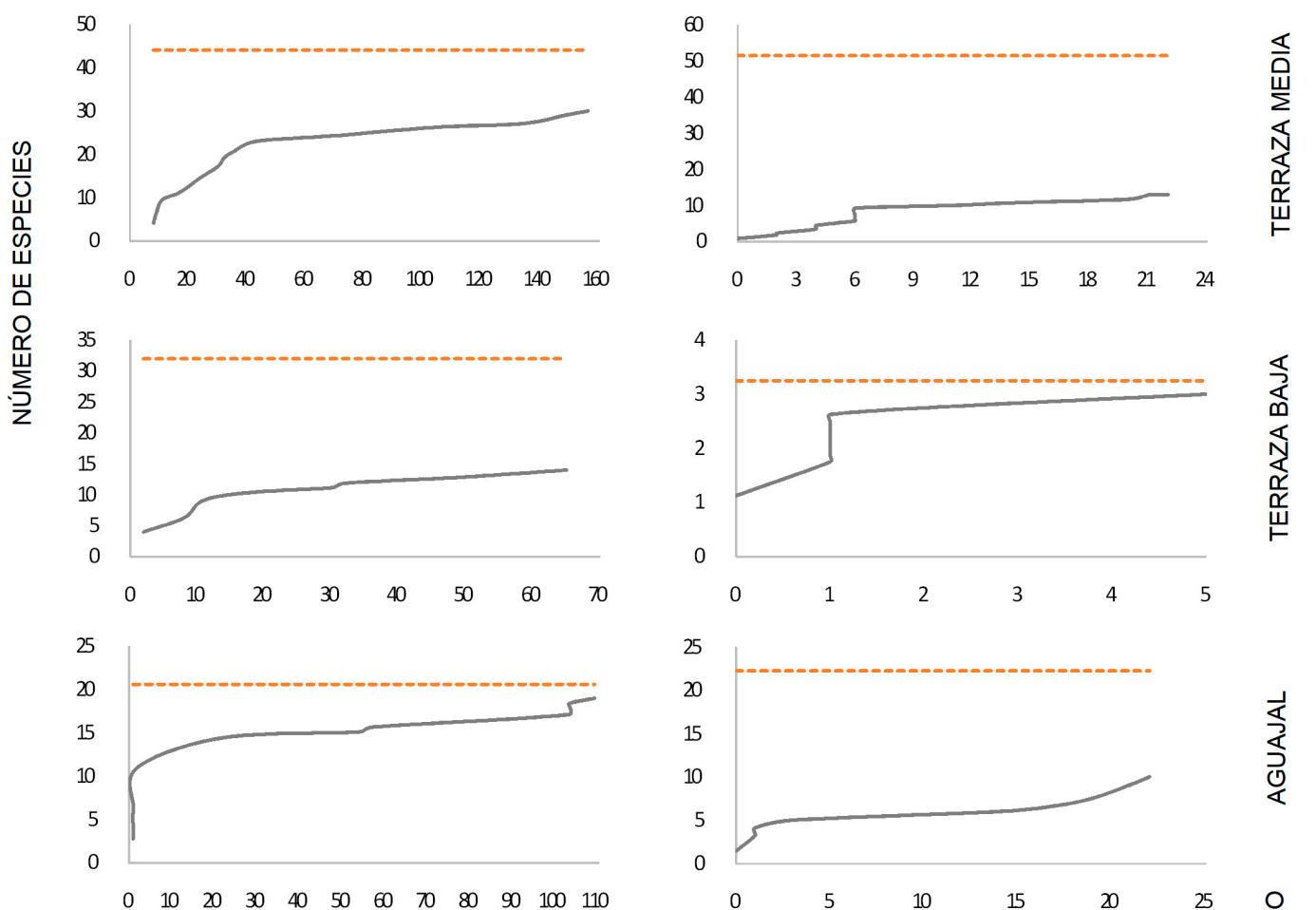

交
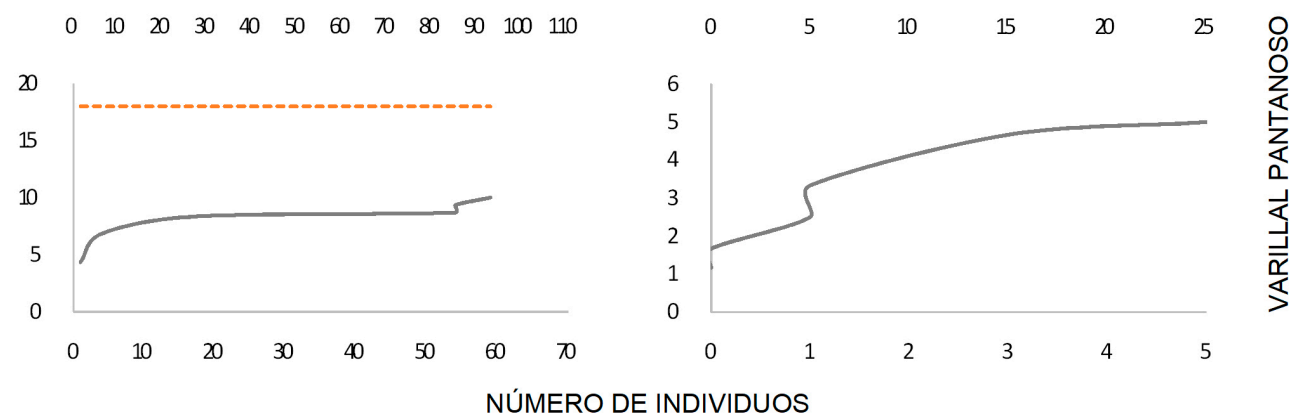

Figura 3: Riqueza de especies de anfibios usando el método de registros por encuentros visuales en los bosques de aguajal, colinas, terraza alta, baja y media y varillal pantanoso en el interfluvio Putumayo-NapoAmazonas. La línea continua presenta la riqueza observada y la línea punteada la riqueza esperada (Chao 1). 
Tabla 2: Listado de especies registradas en el interfluvio Putumayo-Napo-Amazonas desde 1986 al 2019. (1) Yubineto-Ampiyacu (Lescure \& Gasc, 1986); (2) Ampiyacu-Apayacu (Rodríguez \& Knell, 2004); (3) CuyabenoGüeppí (Yáñez-Muñoz \& Venegas, 2008); (4) Maijuna (von May \& Venegas, 2010); (5) Yaguas-Cotuhé (von May \& Mueses-Cisneros, 2011); (6) Lagartococha (Knell, 2012); (7) Ere-Campuya-Algodón (Venegas \& Gagliardi-Urrutia, 2013); (8) Medio Putumayo-Algodón (Chávez \& Mueses-Cisneros, 2016); (9) Alto Putumayo (Pérez-Peña et al., 2019c); (10) Napo-Curaray (Tapia-del Águila et al., 2019); (11) Bajo Putumayo (Tapia-del Águila \& Pérez-Peña, en presa).

\begin{tabular}{|c|c|c|c|c|c|c|c|c|c|c|c|}
\hline \multirow{2}{*}{ Clase/Orden/Familia/Especie } & \multicolumn{11}{|c|}{ Estudios } \\
\hline & 1 & 2 & 3 & 4 & 5 & 6 & 7 & 8 & 9 & 10 & 11 \\
\hline Amphibia (riqueza) & 27 & 58 & 39 & 65 & 67 & 33 & 66 & 86 & 53 & 28 & 45 \\
\hline \multicolumn{12}{|l|}{ Anura } \\
\hline \multicolumn{12}{|l|}{ Aromobatidae } \\
\hline Allobates femoralis & $x$ & $x$ & $x$ & $x$ & $x$ & $x$ & $x$ & $x$ & $x$ & $x$ & $x$ \\
\hline Allobates gr. trilineatus & & & & & & & & & & & $x$ \\
\hline Allobates insperatus & $x$ & $x$ & $x$ & & & $x$ & $x$ & $x$ & $x$ & & \\
\hline Allobates trilineatus & & $x$ & & $x$ & $x$ & & & $x$ & & & \\
\hline \multicolumn{12}{|l|}{ Bufonidae } \\
\hline Amazophrynella amazonicola & $x$ & $x$ & $x$ & $x$ & $x$ & $x$ & $x$ & $x$ & $x$ & & \\
\hline Amazophrynella sp. & & & & & & & & & & & $x$ \\
\hline Amazophrynella siona & & & & & & & & & & $x$ & \\
\hline Atelopus spumarius & $x$ & & & $x$ & & & & & & $x$ & \\
\hline Rhaebo glaberrimus & & $x$ & & & & & & & & & \\
\hline Rhaebo guttatus & & & & & $x$ & & & $x$ & $x$ & & \\
\hline Rhinella castaneotica & & & $x$ & & & & & & $x$ & & \\
\hline Rhinella ceratophrys & & & $x$ & $x$ & $x$ & & $x$ & $x$ & $x$ & & $x$ \\
\hline Rhinella dapsilis & & & $x$ & & $x$ & & $x$ & $x$ & & $x$ & $x$ \\
\hline Rhinella festae & & & & $x$ & & & & & & & \\
\hline Rhinella margaritifera & $x$ & $x$ & $x$ & $x$ & $x$ & $x$ & $x$ & $x$ & $x$ & $x$ & $x$ \\
\hline Rhinella marina & $x$ & $x$ & & $x$ & $x$ & $x$ & $x$ & $x$ & $x$ & & $x$ \\
\hline Rhinella poeppigii & & & & & & & & $x$ & & & \\
\hline Rhinella proboscidea & & & & & & & $x$ & $x$ & $x$ & $x$ & $x$ \\
\hline Rhinella roqueana & & & & & & $x$ & & $x$ & & & $x$ \\
\hline \multicolumn{12}{|l|}{ Centrolenidae } \\
\hline Cochranella resplendens & & & & & & & & $x$ & & & \\
\hline Hyalinobatrachium sp. & & $x$ & & & $x$ & & & $x$ & & & \\
\hline Teratohyla midas & & & & $x$ & $x$ & & $x$ & $x$ & & $x$ & $x$ \\
\hline Vitreorana ritae & & & & & & & & $x$ & & & \\
\hline Ceratophryidae & & & & & & & & $x$ & & & \\
\hline Ceratophrys cornuta & & & & & & & & $x$ & & & \\
\hline \multicolumn{12}{|l|}{ Craugastoridae } \\
\hline Niceforonia nigrovittata & & & & $x$ & $x$ & & $x$ & & & & \\
\hline
\end{tabular}




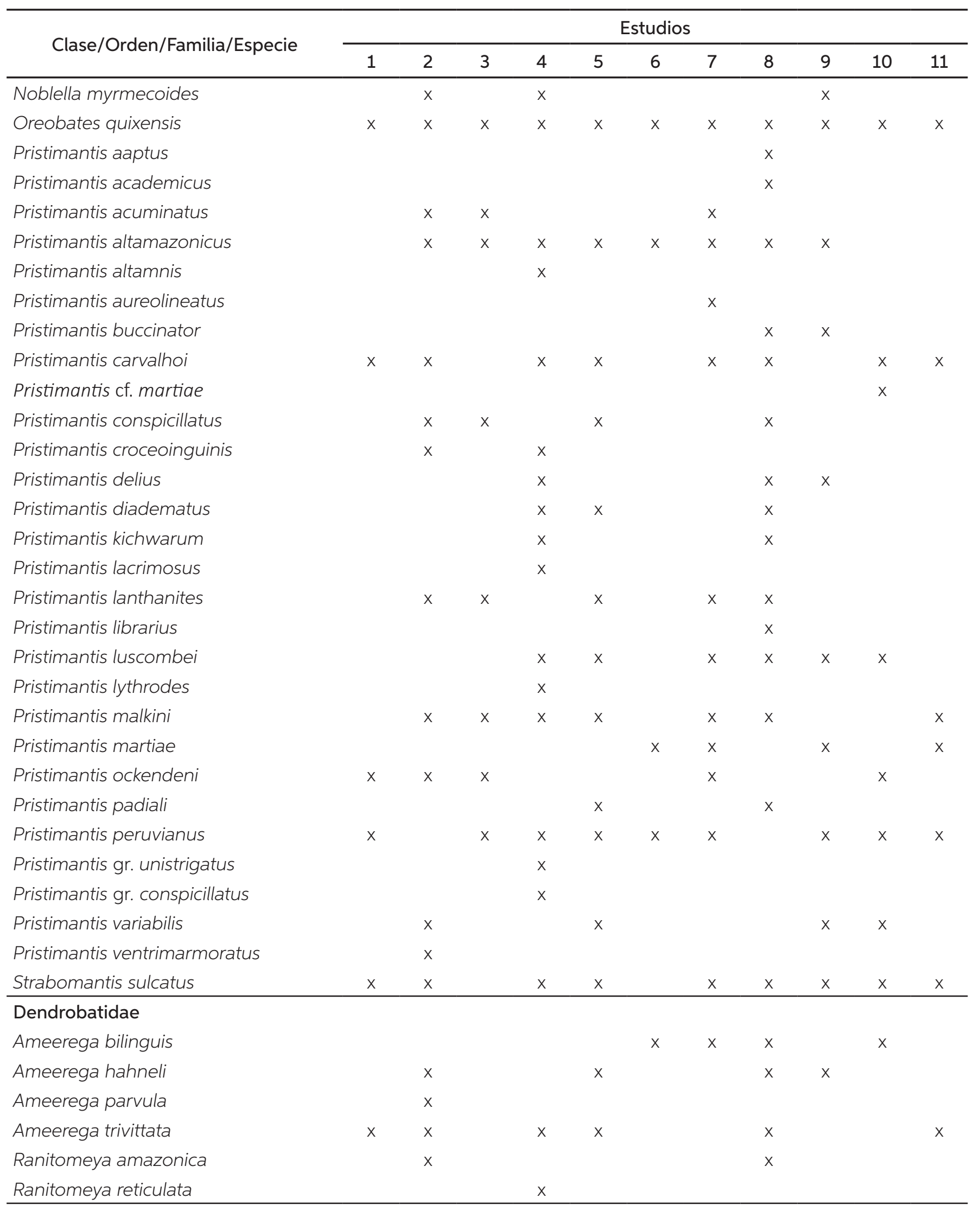




\begin{tabular}{|c|c|c|c|c|c|c|c|c|c|c|c|}
\hline \multirow{2}{*}{ Clase/Orden/Familia/Especie } & \multicolumn{11}{|c|}{ Estudios } \\
\hline & 1 & 2 & 3 & 4 & 5 & 6 & 7 & 8 & 9 & 10 & 11 \\
\hline Ranitomeya variabilis & $x$ & & & & & & $x$ & $x$ & $x$ & & $x$ \\
\hline Ranitomeya ventrimaculata & & & $x$ & $x$ & $x$ & & $x$ & $x$ & & & \\
\hline \multicolumn{12}{|l|}{ Hemiphractidae } \\
\hline Hemiphractus helioi & $x$ & & & & & & & & & & \\
\hline Hemiphractus proboscideus & & & & $x$ & & & & & & & \\
\hline \multicolumn{12}{|l|}{ Hylidae } \\
\hline Boana alfaroi & & & & & & & & & $x$ & & $x$ \\
\hline Boana boans & $x$ & $x$ & $x$ & $x$ & $x$ & $x$ & $x$ & $x$ & $x$ & & \\
\hline Boana calcarata & & $x$ & $x$ & $x$ & $x$ & $x$ & $x$ & $x$ & $x$ & & \\
\hline Boana cinerascens & $x$ & $x$ & $x$ & $x$ & $x$ & $x$ & $x$ & $x$ & $x$ & $x$ & $x$ \\
\hline Boana fasciata & & $x$ & $x$ & $x$ & $x$ & & & & & $x$ & \\
\hline Boana geographica & & $x$ & $x$ & $x$ & $x$ & $x$ & $x$ & $x$ & $x$ & & \\
\hline Boana lanciformis & $x$ & $x$ & $x$ & $x$ & $x$ & $x$ & $x$ & $x$ & $x$ & & $x$ \\
\hline Boana maculateralis & & & & & & & & $x$ & $x$ & & \\
\hline Boana microderma & & & & & $x$ & & & $x$ & & & $x$ \\
\hline Boana nympha & & & $x$ & & $x$ & & & $x$ & & & \\
\hline Boana punctata & & $x$ & & & $x$ & & & & & & \\
\hline Dendropsophus bokermanni & $x$ & & & & & & $x$ & $x$ & & & \\
\hline Dendropsophus brevifrons & & & & & $x$ & & $x$ & $x$ & $x$ & & \\
\hline Dendropsophus koechlini & & & & $x$ & & & & & & & \\
\hline Dendropsophus marmoratus & $x$ & $x$ & & $x$ & $x$ & & $x$ & $x$ & & & $x$ \\
\hline Dendropsophus parviceps & & & & & $x$ & & & $x$ & & & $x$ \\
\hline Dendropsophus rhodopeplus & & & & $x$ & & & $x$ & $x$ & & & \\
\hline Dendropsophus riveroi & & & & & & & & & $x$ & & \\
\hline Dendropsophus sarayacuensis & & & & & & & $x$ & $x$ & & & $x$ \\
\hline Dendropsophus triangulum & & & $x$ & & $x$ & $x$ & & $x$ & & & \\
\hline Hyloscirtus albopunctulatus & & $x$ & & & & & & & & & \\
\hline Nyctimantis rugiceps & & & $x$ & $x$ & $x$ & $x$ & $x$ & & $x$ & & $x$ \\
\hline Osteocephalus buckleyi & & $x$ & & & & & & & & & \\
\hline Osteocephalus cabrerai & & $x$ & $x$ & $x$ & $x$ & & & $x$ & & & \\
\hline Osteocephalus deridens & & $x$ & & & $x$ & $x$ & $x$ & $x$ & $x$ & & $x$ \\
\hline Osteocephalus fuscifacies & & & $x$ & $x$ & & & & $x$ & & & \\
\hline Osteocephalus heyeri & & & & & $x$ & & & $x$ & & & \\
\hline Osteocephalus mutabor & & $x$ & & & $x$ & $x$ & $x$ & $x$ & $x$ & $x$ & \\
\hline Osteocephalus planiceps & & $x$ & $x$ & $x$ & $x$ & $x$ & $x$ & $x$ & $x$ & $x$ & $x$ \\
\hline Osteocephalus taurinus & & $x$ & $x$ & $x$ & $x$ & $x$ & $x$ & $x$ & $x$ & & $x$ \\
\hline Osteocephalus yasuni & & $x$ & & $x$ & $x$ & $x$ & $x$ & $x$ & $x$ & & \\
\hline Scinax cruentomma & & $x$ & & & $x$ & $x$ & & $x$ & & & \\
\hline
\end{tabular}




\begin{tabular}{|c|c|c|c|c|c|c|c|c|c|c|c|}
\hline \multirow{2}{*}{ Clase/Orden/Familia/Especie } & \multicolumn{11}{|c|}{ Estudios } \\
\hline & 1 & 2 & 3 & 4 & 5 & 6 & 7 & 8 & 9 & 10 & 11 \\
\hline Scinax garbei & & & & & $x$ & $x$ & & $x$ & $x$ & & $x$ \\
\hline Scinax ruber & & & & & & & $x$ & & $x$ & & $x$ \\
\hline Sphaenorhynchus dorisae & & & & & & $x$ & & & & & \\
\hline Tepuihyla tuberculosa & & & & & & & $x$ & $x$ & $x$ & & $x$ \\
\hline Trachycephalus cunauaru & & $x$ & $x$ & $x$ & $x$ & $x$ & $x$ & $x$ & & & \\
\hline Trachycephalus typhonius & & & & & & & & & $x$ & $x$ & $x$ \\
\hline \multicolumn{12}{|l|}{ Leptodactylidae } \\
\hline Adenomera andreae & $x$ & $x$ & $x$ & $x$ & $x$ & $x$ & $x$ & $x$ & $x$ & $x$ & $x$ \\
\hline Edalorhina perezi & $x$ & $x$ & $x$ & $x$ & $x$ & & $x$ & $x$ & & $x$ & $x$ \\
\hline Engystomops petersi & $x$ & $x$ & & $x$ & $x$ & & $x$ & $x$ & $x$ & $x$ & \\
\hline Leptodactylus diedrus & & & & $x$ & & & $x$ & $x$ & & & $x$ \\
\hline Leptodactylus discodactylus & & & $x$ & & $x$ & & $x$ & & $x$ & & \\
\hline Leptodactylus knudseni & & & $x$ & $x$ & & & $x$ & $x$ & & & \\
\hline Leptodactylus leptodactyloides & & $x$ & & & $x$ & & & & $x$ & & $x$ \\
\hline Leptodactylus mystaceus & & & & & & $x$ & & & $x$ & & \\
\hline Leptodactylus pentadactylus & $x$ & $x$ & $x$ & $x$ & $x$ & $x$ & $x$ & $x$ & $x$ & $x$ & $x$ \\
\hline Leptodactylus petersii & & $x$ & & $x$ & $x$ & & $x$ & $x$ & & & $x$ \\
\hline Leptodactylus rhodomystax & $x$ & & $x$ & $x$ & & & $x$ & $x$ & & & $x$ \\
\hline Leptodactylus stenodema & & & & & & & $x$ & & & & $x$ \\
\hline Leptodactylus wagneri & $x$ & & $x$ & $x$ & $x$ & $x$ & $x$ & $x$ & $x$ & & \\
\hline Lithodytes lineatus & $x$ & $x$ & $x$ & $x$ & $x$ & & $x$ & $x$ & $x$ & & \\
\hline \multicolumn{12}{|l|}{ Microhylidae } \\
\hline Chiasmocleis anatipes & & $x$ & & & & & & & & & \\
\hline Chiasmocleis antenori & & $x$ & & & & $x$ & & & & & \\
\hline Chiasmocleis bassleri & & $x$ & & $x$ & $x$ & & $x$ & $x$ & $x$ & & $x$ \\
\hline Chiasmocleis carvalhoi & & & & $x$ & & & $x$ & $x$ & $x$ & $x$ & \\
\hline Chiasmocleis magnova & & & & & & & $x$ & & & & \\
\hline Chiasmocleis tridactyla & & & & & $x$ & & & & & & $x$ \\
\hline Chiasmocleis ventrimaculata & & & & & & & $x$ & $x$ & & & \\
\hline Hamptophryne boliviana & & $x$ & & & & & $x$ & & & & \\
\hline Synapturanus rabus & & & & & $x$ & & $x$ & $x$ & & & \\
\hline \multicolumn{12}{|l|}{ Phyllomedusidae } \\
\hline Callimedusa atelopoides & & $x$ & & & & & & & & & \\
\hline Callimedusa tomopterna & & & & $x$ & & & $x$ & $x$ & $x$ & & \\
\hline Phyllomedusa bicolor & & & & $x$ & $x$ & & & $x$ & & & $x$ \\
\hline Phyllomedusa tarsius & & & $x$ & & & & $x$ & $x$ & $x$ & & \\
\hline Phyllomedusa vaillantii & & $x$ & & $x$ & $x$ & & $x$ & $x$ & & & $x$ \\
\hline Pithecopus palliatus & & & & & & & $x$ & & & & \\
\hline
\end{tabular}




\section{Clase/Orden/Familia/Especie}

\section{Estudios}

$\begin{array}{lllllllllll}1 & 2 & 3 & 4 & 5 & 6 & 7 & 8 & 9 & 10 & 11\end{array}$

Pipidae

Pipa pipa

$x$

$X$

$X$

Caudata

\section{Plethodontidae}

Bolitoglossa altamazonica

$x \quad x \quad x$

$\mathrm{X}$

Bolitoglossa equatoriana

Bolitoglossa peruviana

$x$

Gymnophiona

\section{Caeciliidae}

Caecilia sp.

Oscaecilia sp.

$x+x$

Siphonops annulatus

Reptilia (Riqueza)

$x$

Crocodylia

\section{Alligatoridae}

Caiman crocodilus

Melanosuchus niger

Paleosuchus trigonatus

$\begin{array}{lllllllllll}25 & 40 & 34 & 42 & 52 & 21 & 59 & 52 & 32 & 16 & 32\end{array}$

Squamata

Alopoglossidae

Alopoglossus angulatus

Alopoglossus atriventris

Alopoglossus brevifrontalis

Alopoglossus buckleyi

$x \quad x$

$x \quad x$

$x$

$x \quad x$

X $\quad x$

X

$x \quad x \quad x$

$x \quad x$

$\mathrm{X}$

Alopoglossus copii

Amphisbaenidae

Amphisbaena fuliginosa

\section{Boidae}

Boa constrictor

Corallus batesii

Corallus hortulanus

Epicrates cenchria

Eunectes murinus

\begin{tabular}{|c|c|c|c|c|c|c|c|c|c|}
\hline$x$ & & & $x$ & & & $x$ & & $x$ & \\
\hline & $x$ & $x$ & $x$ & $x$ & $x$ & $X$ & $x$ & $x$ & $x$ \\
\hline$x$ & $x$ & $x$ & $x$ & & & $X$ & & & \\
\hline$X$ & & & $X$ & & & & & & \\
\hline
\end{tabular}

\section{Colubridae}

Atractus collaris

Atractus gaigeae

Atractus major

Atractus snethlageae

$x-x$

$x$ 


\begin{tabular}{|c|c|c|c|c|c|c|c|c|c|c|c|}
\hline \multirow{2}{*}{ Clase/Orden/Familia/Especie } & \multicolumn{11}{|c|}{ Estudios } \\
\hline & 1 & 2 & 3 & 4 & 5 & 6 & 7 & 8 & 9 & 10 & 11 \\
\hline Atractus torquatus & & & & & & & $x$ & $x$ & & & \\
\hline Chironius fuscus & & $x$ & & $x$ & $x$ & $x$ & $x$ & $x$ & $x$ & & $x$ \\
\hline Clelia clelia & & $x$ & $x$ & $x$ & $x$ & & $x$ & & & & \\
\hline Dendrophidion dendrophis & & & & & & & $x$ & & & & \\
\hline Dipsas catesbyi & & & $x$ & & & & $x$ & & & & \\
\hline Dipsas indica & & & & & & & $x$ & & & & \\
\hline Drepanoides anomalus & & & $x$ & & $x$ & & $x$ & & & & $x$ \\
\hline Drymarchon corais & & & & & $x$ & & & & & & \\
\hline Drymoluber dichrous & & & & $x$ & & & & & & & \\
\hline Erythrolamprus epinephalus & & & & & $x$ & & $x$ & & & & \\
\hline Erythrolamprus mimus & & & & & & & $x$ & $x$ & & & \\
\hline Erythrolamprus reginae & & & & & $x$ & & & & & & \\
\hline Erythrolamprus sp. & & & & & & & & & $x$ & & \\
\hline Erythrolamprus typhlus & & & & & & & $x$ & $x$ & & & \\
\hline Helicops angulatus & & & & & & $x$ & $x$ & & $x$ & & $x$ \\
\hline Helicops polylepis & & & & & $x$ & & & & & & \\
\hline Hydrops martii & & & $x$ & & $x$ & & & & & & \\
\hline Imantodes cenchoa & & $x$ & $x$ & $x$ & $x$ & $x$ & $x$ & $x$ & $x$ & & $x$ \\
\hline Imantodes lentiferus & & & & & & & & & $x$ & & \\
\hline Leptodeira annulata & & $x$ & & $x$ & $x$ & & $x$ & & $x$ & $x$ & \\
\hline Leptophis ahaetulla & & & & & & $x$ & & $x$ & $x$ & & \\
\hline Oxybelis fulgidus & & & & $x$ & & & & & $x$ & & \\
\hline Oxyrhopus formosus & & & $x$ & & $x$ & & $x$ & & & & $x$ \\
\hline Oxyrhopus melanogenys & & & $x$ & & $x$ & & & & & $x$ & \\
\hline Oxyrhopus petolarius & & & $x$ & & & & $x$ & & $x$ & & $x$ \\
\hline Philodryas argentea & & $x$ & & $x$ & & & $x$ & $x$ & & & $x$ \\
\hline Phrynonax poecilonotus & & & & $x$ & & & $x$ & & $x$ & & \\
\hline Phrynonax polylepis & & & & & & & & $x$ & & & \\
\hline Pseudoboa coronata & & $x$ & & & $x$ & & $x$ & $x$ & $x$ & & $x$ \\
\hline Rhinobothryum lentiginosum & & $x$ & & & & & & & & & \\
\hline Siphlophis compressus & & & & $x$ & & & $x$ & $x$ & $x$ & $x$ & $x$ \\
\hline Spilotes pullatus & & & & & & & $x$ & & & & \\
\hline Taeniophallus brevirostris & & $x$ & & & $x$ & & & & & & \\
\hline Xenodon rabdocephalus & & $x$ & & & $x$ & & & $x$ & $x$ & $x$ & \\
\hline Xenopholis scalaris & & $x$ & & & $x$ & & & $\mathrm{x}$ & $\mathrm{x}$ & & $\mathrm{x}$ \\
\hline
\end{tabular}

\section{Dactyloidae}

Anolis bombiceps 


\begin{tabular}{|c|c|c|c|c|c|c|c|c|c|c|c|}
\hline \multirow{2}{*}{ Clase/Orden/Familia/Especie } & \multicolumn{11}{|c|}{ Estudios } \\
\hline & 1 & 2 & 3 & 4 & 5 & 6 & 7 & 8 & 9 & 10 & 11 \\
\hline Anolis ortonii & $x$ & $x$ & $x$ & $x$ & $x$ & $x$ & & $x$ & & & \\
\hline Anolis punctatus & $x$ & $x$ & & $x$ & $x$ & $x$ & & $x$ & & & \\
\hline Anolis scypheus & $x$ & $x$ & $x$ & $x$ & $x$ & & $x$ & $x$ & $x$ & & $x$ \\
\hline Anolis trachyderma & $x$ & $x$ & & $x$ & $x$ & $x$ & $x$ & $x$ & $x$ & & $x$ \\
\hline Anolis transversalis & $x$ & $x$ & & $x$ & $x$ & & $x$ & $x$ & $x$ & & \\
\hline \multicolumn{12}{|l|}{ Elapidae } \\
\hline Micrurus annellatus & & & & & & & & $x$ & & & \\
\hline Micrurus filiformis & & & & & & & & & & $x$ & \\
\hline Micrurus hemprichii & & & & $x$ & & & $x$ & $x$ & & $x$ & \\
\hline Micrurus langsdorffi & & $x$ & & $x$ & $x$ & & $x$ & & & & \\
\hline Micrurus lemniscatus & & $x$ & $x$ & & $x$ & & $x$ & $x$ & & & \\
\hline Micrurus obscurus & & & & & & & & $x$ & & & \\
\hline Micrurus putumayensis & & & & & $x$ & & & & & & \\
\hline Micrurus surinamensis & & & $x$ & & & & & & & & \\
\hline \multicolumn{12}{|l|}{ Gymnophthalmidae } \\
\hline Arthrosaura reticulata & & & $x$ & & $x$ & $x$ & & $x$ & $x$ & & \\
\hline Bachia trisanale & & & & & & & $x$ & & & & \\
\hline Cercosaura argula & $x$ & $x$ & $x$ & $x$ & $x$ & $x$ & $x$ & $x$ & $x$ & $x$ & $x$ \\
\hline Cercosaura ocellata & $x$ & & & & & & & & & & \\
\hline Cercosaura oshaughnessyi & & & & & & & & $x$ & & $x$ & \\
\hline Iphisa elegans & $x$ & & & & & & $x$ & $x$ & & & \\
\hline Loxopholis parietalis & $x$ & & $x$ & & & & $x$ & & $x$ & & \\
\hline Potamites ecpleopus & $x$ & $x$ & $x$ & $x$ & $x$ & & $x$ & $x$ & & & \\
\hline \multicolumn{12}{|l|}{ Hoplocercidae } \\
\hline Enyalioides laticeps & $x$ & $x$ & $x$ & $x$ & & & $x$ & $x$ & & $x$ & \\
\hline \multicolumn{12}{|l|}{ Leptotyphlopidae } \\
\hline Epictia sp. & & & & & & & $x$ & & & & \\
\hline \multicolumn{12}{|l|}{ Phyllodactylidae } \\
\hline Techadactylus solimoensis & & & $x$ & & $x$ & & $x$ & $x$ & & & $x$ \\
\hline \multicolumn{12}{|l|}{ Polychrotidae } \\
\hline Polychrus marmoratus & $x$ & & & & & & & & & & \\
\hline \multicolumn{12}{|l|}{ Scincidae } \\
\hline Copeoglossum nigropunctatum & $x$ & & $x$ & $x$ & $x$ & & & & $x$ & & \\
\hline Varzea altamazonica & & & & & & & $x$ & & & & $x$ \\
\hline \multicolumn{12}{|l|}{ Sphaerodactylidae } \\
\hline Gonatodes concinnatus & $x$ & $x$ & $x$ & $x$ & $x$ & & $x$ & & & & \\
\hline Gonatodes humeralis & $x$ & $x$ & $x$ & $x$ & $x$ & $x$ & $x$ & $x$ & $x$ & $x$ & $x$ \\
\hline Lepidoblepharis hoogmoedi & & $x$ & & & & & & & & & \\
\hline
\end{tabular}




\begin{tabular}{|c|c|c|c|c|c|c|c|c|c|c|c|}
\hline \multirow{2}{*}{ Clase/Orden/Familia/Especie } & \multicolumn{11}{|c|}{ Estudios } \\
\hline & 1 & 2 & 3 & 4 & 5 & 6 & 7 & 8 & 9 & 10 & 11 \\
\hline Pseudogonatodes guianensis & & $x$ & & & $x$ & & $x$ & $x$ & & & \\
\hline \multicolumn{12}{|l|}{ Teiidae } \\
\hline Ameiva ameiva & $x$ & & & $x$ & & $x$ & & & $x$ & & \\
\hline Kentropyx altamazonica & & & & & & $x$ & & & & & $x$ \\
\hline Kentropyx pelviceps & $x$ & $x$ & $x$ & $x$ & $x$ & & $x$ & $x$ & $x$ & & $x$ \\
\hline Tupinambis teguixin & $x$ & $x$ & & $x$ & $x$ & $x$ & $x$ & $x$ & & & \\
\hline \multicolumn{12}{|l|}{ Tropiduridae } \\
\hline Plica plica & & $x$ & & $x$ & $x$ & & & & & & $x$ \\
\hline Plica umbra & $x$ & $x$ & $x$ & $x$ & $x$ & & $x$ & $x$ & $x$ & $x$ & $x$ \\
\hline Uracentron azureum & & & & & & & & $x$ & & & \\
\hline Uracentron flaviceps & $x$ & & & $x$ & & & & & & & \\
\hline \multicolumn{12}{|l|}{ Typhlopidae } \\
\hline Amerotyphlops minuisquamus & & & & & & & & $x$ & & & \\
\hline \multicolumn{12}{|l|}{ Viperidae } \\
\hline Bothrocophias hyoprora & & & $x$ & $x$ & & & $x$ & $x$ & $x$ & $x$ & \\
\hline Bothrops atrox & & $x$ & & $x$ & $x$ & & $x$ & $x$ & & $x$ & \\
\hline Bothrops bilineatus & & & & & $x$ & & & & & & \\
\hline Bothrops brazili & & & & & & & $x$ & & & & \\
\hline Bothrops taeniatus & & & & & & & $x$ & $x$ & & & \\
\hline Lachesis muta & & & & $x$ & & & & $x$ & & & \\
\hline \multicolumn{12}{|l|}{ Testudines } \\
\hline \multicolumn{12}{|l|}{ Chelidae } \\
\hline Chelus fimbriata & & $x$ & & & $x$ & & $x$ & & & & \\
\hline Mesoclemmys gibba & & & & & $x$ & & $x$ & $x$ & & & \\
\hline Mesoclemys sp. & & & & & & & & & & & $x$ \\
\hline \multicolumn{12}{|l|}{ Podocnemididae } \\
\hline Podocnemis expansa & & & & & & & $x$ & $x$ & $x$ & & \\
\hline Podocnemis sextuberculata & & $x$ & & & $x$ & & & & & & \\
\hline Podocnemis unifilis & & & & & & $x$ & & $x$ & & & $x$ \\
\hline \multicolumn{12}{|l|}{ Testudinidae } \\
\hline Chelonoidis denticulata & & $x$ & & $x$ & $x$ & $x$ & $x$ & $x$ & & & $x$ \\
\hline Riqueza Total & 52 & 98 & 73 & 107 & 119 & 54 & 125 & 138 & 85 & 44 & 77 \\
\hline
\end{tabular}


Tabla 3. Lista de especies de anfibios y reptiles por tipo de hábitats en el interfluvio Putumayo-Napo-Amazonas.

\begin{tabular}{|c|c|c|c|c|c|c|}
\hline Clase/orden/familia/especie & Aguajal & Colina baja & Terraza alta & $\begin{array}{c}\text { Terraza } \\
\text { baja }\end{array}$ & $\begin{array}{c}\text { Terraza } \\
\text { media }\end{array}$ & $\begin{array}{c}\text { Varillal } \\
\text { pantanoso }\end{array}$ \\
\hline Amphibia (riqueza) & 19 & 58 & 15 & 14 & 30 & 10 \\
\hline \multicolumn{7}{|l|}{ Anura } \\
\hline \multicolumn{7}{|l|}{ Aromobatidae } \\
\hline Allobates femoralis & $x$ & $x$ & $x$ & & $x$ & $x$ \\
\hline Allobates gr. trilineatus & & & & & $x$ & \\
\hline Allobates insperatus & $x$ & $x$ & & & & \\
\hline \multicolumn{7}{|l|}{ Bufonidae } \\
\hline Amazophrynella amazonicola & $x$ & $x$ & & $x$ & & \\
\hline Amazophrynella sp. & & $x$ & $x$ & & & \\
\hline Amazophrynella siona & & $x$ & & & & \\
\hline Atelopus spumarius & & $x$ & & & & \\
\hline Rhinella castaneotica & & $x$ & & & & \\
\hline Rhinella ceratophrys & & $x$ & & & & \\
\hline Rhinella dapsilis & & $x$ & & & & \\
\hline Rhinella margaritifera & $x$ & $x$ & $x$ & $x$ & $x$ & \\
\hline Rhinella marina & & $x$ & & & & $x$ \\
\hline Rhinella proboscidea & $x$ & $x$ & $x$ & & $x$ & \\
\hline Rhinella roqueana & & $x$ & & & & \\
\hline \multicolumn{7}{|l|}{ Centrolenidae } \\
\hline Teratohyla midas & & $x$ & & & & \\
\hline \multicolumn{7}{|l|}{ Craugastoridae } \\
\hline Noblella myrmecoides & & $x$ & & & & \\
\hline Oreobates quixensis & $x$ & $x$ & & & $x$ & \\
\hline Pristimantis altamazonicus & $x$ & & & & & \\
\hline Pristimantis buccinator & & $x$ & & & & \\
\hline Pristimantis carvalhoi & & $x$ & $x$ & & $x$ & \\
\hline Pristimantis cf. martiae & & $x$ & & & & \\
\hline Pristimantis delius & & $x$ & & & & \\
\hline Pristimantis luscombei & & $x$ & & & & \\
\hline Pristimantis malkini & & $x$ & & & $x$ & \\
\hline Pristimantis martiae & & $x$ & $x$ & & & \\
\hline Pristimantis ockendeni & & $x$ & & & & \\
\hline Pristimantis peruvianus & & $x$ & $x$ & & $x$ & \\
\hline Pristimantis variabilis & & $x$ & & & & \\
\hline Strabomantis sulcatus & & $x$ & & & $x$ & \\
\hline
\end{tabular}




\begin{tabular}{|c|c|c|c|c|c|c|}
\hline Clase/orden/familia/especie & Aguajal & Colina baja & Terraza alta & $\begin{array}{c}\text { Terraza } \\
\text { baja }\end{array}$ & $\begin{array}{c}\text { Terraza } \\
\text { media }\end{array}$ & $\begin{array}{c}\text { Varillal } \\
\text { pantanoso }\end{array}$ \\
\hline \multicolumn{7}{|l|}{ Dendrobatidae } \\
\hline Ameerega bilinguis & & $x$ & & & & \\
\hline Ameerega hahneli & $x$ & & & & & \\
\hline Ameerega trivittata & & & & & $x$ & \\
\hline Ranitomeya variabilis & $x$ & & & $x$ & $x$ & $x$ \\
\hline \multicolumn{7}{|l|}{ Hylidae } \\
\hline Boana alfaroi & $x$ & $x$ & $x$ & & $x$ & \\
\hline Boana calcarata & & $x$ & & & & \\
\hline Boana cinerascens & & $x$ & $x$ & $x$ & & $x$ \\
\hline Boana fasciata & & $x$ & & & & \\
\hline Boana geographica & $x$ & $x$ & & $x$ & & $x$ \\
\hline Boana lanciformis & $x$ & $x$ & & $x$ & $x$ & $x$ \\
\hline Boana maculateralis & & $x$ & & & & \\
\hline Boana microderma & & & & & $x$ & \\
\hline Dendropsophus brevifrons & $x$ & & & & & \\
\hline Dendropsophus marmoratus & & $x$ & & & $x$ & \\
\hline Dendropsophus parviceps & & $x$ & & & & \\
\hline Dendropsophus riveroi & $x$ & & & $x$ & & \\
\hline Dendropsophus sarayacuensis & & $x$ & & & & \\
\hline Nyctimantis rugiceps & & $x$ & & & & \\
\hline Osteocephalus deridens & & & & $x$ & $x$ & \\
\hline Osteocephalus mutabor & & $x$ & & & & \\
\hline Osteocephalus planiceps & $x$ & $x$ & $x$ & $x$ & $x$ & $x$ \\
\hline Osteocephalus taurinus & & $x$ & & $x$ & & \\
\hline Osteocephalus yasuni & $x$ & & & $x$ & & \\
\hline Scinax garbei & & & & & $x$ & \\
\hline Scinax ruber & & & & & $x$ & \\
\hline Tepuihyla tuberculosa & & $x$ & & & & \\
\hline Trachycephalus typhonius & $x$ & $x$ & & $x$ & $x$ & $x$ \\
\hline \multicolumn{7}{|l|}{ Leptodactylidae } \\
\hline Adenomera andreae & $x$ & $x$ & & & $x$ & \\
\hline Edalorhina perezi & & $x$ & & & & \\
\hline Engystomops petersi & & $x$ & & & & \\
\hline Leptodactylus diedrus & & $x$ & & & $x$ & \\
\hline Leptodactylus discodactylus & $x$ & $x$ & & $x$ & & $x$ \\
\hline Leptodactylus leptodactyloides & & $x$ & $x$ & & $x$ & \\
\hline Leptodactylus pentadactylus & & $x$ & & & $x$ & \\
\hline Leptodactylus petersii & & & & & $x$ & \\
\hline
\end{tabular}




\begin{tabular}{|c|c|c|c|c|c|c|}
\hline Clase/orden/familia/especie & Aguajal & Colina baja & Terraza alta & $\begin{array}{c}\text { Terraza } \\
\text { baja }\end{array}$ & $\begin{array}{l}\text { Terraza } \\
\text { media }\end{array}$ & $\begin{array}{c}\text { Varillal } \\
\text { pantanoso }\end{array}$ \\
\hline Leptodactylus rhodomystax & & & $x$ & & $x$ & \\
\hline Leptodactylus stenodema & & & $x$ & & $x$ & \\
\hline Leptodactylus wagneri & & $x$ & & & & \\
\hline \multicolumn{7}{|l|}{ Microhylidae } \\
\hline Chiasmocleis bassleri & & $x$ & $x$ & & $x$ & \\
\hline Chiasmocleis carvalhoi & & $x$ & & & & \\
\hline Chiasmocleis tridactyla & & & & & $x$ & \\
\hline \multicolumn{7}{|l|}{ Phyllomedusidae } \\
\hline Callimedusa tomopterna & & $x$ & & $x$ & & \\
\hline Phyllomedusa bicolor & & & & & $x$ & \\
\hline Phyllomedusa tarsius & & $x$ & & & & \\
\hline Phyllomedusa vaillantii & & & $x$ & & & \\
\hline
\end{tabular}

\section{Pipidae}

Pipa pipa

Caudata

\section{Plethodontidae}

Bolitoglossa equatoriana $\quad x$

Bolitoglossa peruviana

\begin{tabular}{|c|c|c|c|c|c|c|}
\hline Reptilia (riqueza) & 10 & 35 & 11 & 5 & 12 & 5 \\
\hline \multicolumn{7}{|l|}{ Crocodylia } \\
\hline \multicolumn{7}{|l|}{ Alligatoridae } \\
\hline Caiman crocodilus & & & & $x$ & & \\
\hline Melanosuchus niger & & & & $x$ & & \\
\hline Paleosuchus trigonatus & & $x$ & & & $x$ & \\
\hline
\end{tabular}

Squamata

Alopoglossidae

Alopoglossus angulatus

$x \quad x$

Alopoglossus atriventris

$x$

$x$

Alopoglossus copii

\section{Boidae}

Corallus hortulanus

Epicrates cenchria

Eunectes murinus

\section{Colubridae}

Chironius fuscus

Drepanoides anomalus

Erythrolamprus sp.

Helicops angulatus
$X$

$\mathrm{X}$

X

X

$X$

$x-x$

X 


\begin{tabular}{|c|c|c|c|c|c|c|}
\hline Clase/orden/familia/especie & Aguajal & Colina baja & Terraza alta & $\begin{array}{c}\text { Terraza } \\
\text { baja }\end{array}$ & $\begin{array}{c}\text { Terraza } \\
\text { media }\end{array}$ & $\begin{array}{c}\text { Varillal } \\
\text { pantanoso }\end{array}$ \\
\hline Imantodes cenchoa & & $x$ & & & & $x$ \\
\hline Imantodes lentiferus & & $x$ & & & & \\
\hline Leptodeira annulata & & $x$ & & $x$ & & \\
\hline Leptophis ahaetulla & & $x$ & & & & \\
\hline Oxybelis fulgidus & $x$ & & & & & \\
\hline Oxyrhopus formosus & & & & & $x$ & \\
\hline Oxyrhopus melanogenys & & $x$ & & & & \\
\hline Oxyrhopus petolarius & & $x$ & & & & \\
\hline Philodryas argentea & & $x$ & & & & \\
\hline Phrynonax poecilonotus & & $x$ & & & & \\
\hline Pseudoboa coronata & $x$ & $x$ & & & & \\
\hline Siphlophis compressus & $x$ & $x$ & $x$ & & & \\
\hline Xenodon rabdocephalus & & $x$ & $x$ & & & \\
\hline Xenopholis scalaris & & $x$ & $x$ & & & \\
\hline \multicolumn{7}{|l|}{ Dactyloidae } \\
\hline Anolis fuscoauratus & & & & & $x$ & \\
\hline Anolis scypheus & & $x$ & $x$ & & & \\
\hline Anolis trachyderma & & $x$ & $x$ & & $x$ & $x$ \\
\hline Anolis transversalis & & $x$ & & & & \\
\hline \multicolumn{7}{|l|}{ Elapidae } \\
\hline Micrurus filiformis & & $x$ & & & & \\
\hline Micrurus hemprichii & & $x$ & & & & \\
\hline \multicolumn{7}{|l|}{ Gymnophthalmidae } \\
\hline Arthrosaura reticulata & & $x$ & & & & \\
\hline Cercosaura argula & & $x$ & & & & \\
\hline Cercosaura oshaughnessyi & & $x$ & & & & \\
\hline Loxopholis parietalis & & $x$ & & & & \\
\hline \multicolumn{7}{|l|}{ Hoplocercidae } \\
\hline Enyalioides laticeps & & $x$ & & & & \\
\hline \multicolumn{7}{|l|}{ Phyllodactylidae } \\
\hline Techadactylus solimoensis & & & & & $x$ & \\
\hline \multicolumn{7}{|l|}{ Scincidae } \\
\hline Copeoglossum nigropunctatum & $x$ & & & & & \\
\hline Varzea altamazonica & & $x$ & & & & \\
\hline \multicolumn{7}{|l|}{ Sphaerodactylidae } \\
\hline Gonatodes humeralis & $\mathrm{x}$ & $x$ & $\mathrm{x}$ & & & \\
\hline
\end{tabular}




\begin{tabular}{|c|c|c|c|c|c|c|}
\hline Clase/orden/familia/especie & Aguajal & Colina baja & Terraza alta & $\begin{array}{c}\text { Terraza } \\
\text { baja }\end{array}$ & $\begin{array}{c}\text { Terraza } \\
\text { media }\end{array}$ & $\begin{array}{c}\text { Varillal } \\
\text { pantanoso }\end{array}$ \\
\hline \multicolumn{7}{|l|}{ Teiidae } \\
\hline Kentropyx altamazonica & & & $x$ & & & \\
\hline Kentropyx pelviceps & $x$ & $x$ & $x$ & $x$ & $x$ & $x$ \\
\hline \multicolumn{7}{|l|}{ Tropiduridae } \\
\hline Plica plica & & $x$ & & & & \\
\hline Plica umbra & $x$ & $x$ & $x$ & & & \\
\hline \multicolumn{7}{|l|}{ Viperidae } \\
\hline Bothrocophias hyoprora & $x$ & $x$ & & & & \\
\hline Bothrops atrox & & $x$ & & & & \\
\hline \multicolumn{7}{|l|}{ Testudines } \\
\hline \multicolumn{7}{|l|}{ Chelidae } \\
\hline Mesoclemys sp. & & $x$ & & & & \\
\hline \multicolumn{7}{|l|}{ Podocnemididae } \\
\hline Podocnemis unifilis & & & & $x$ & & \\
\hline \multicolumn{7}{|l|}{ Testudinidae } \\
\hline Chelonoidis denticulata & & $x$ & & & $x$ & \\
\hline Total & 29 & 93 & 26 & 19 & 42 & 15 \\
\hline
\end{tabular}




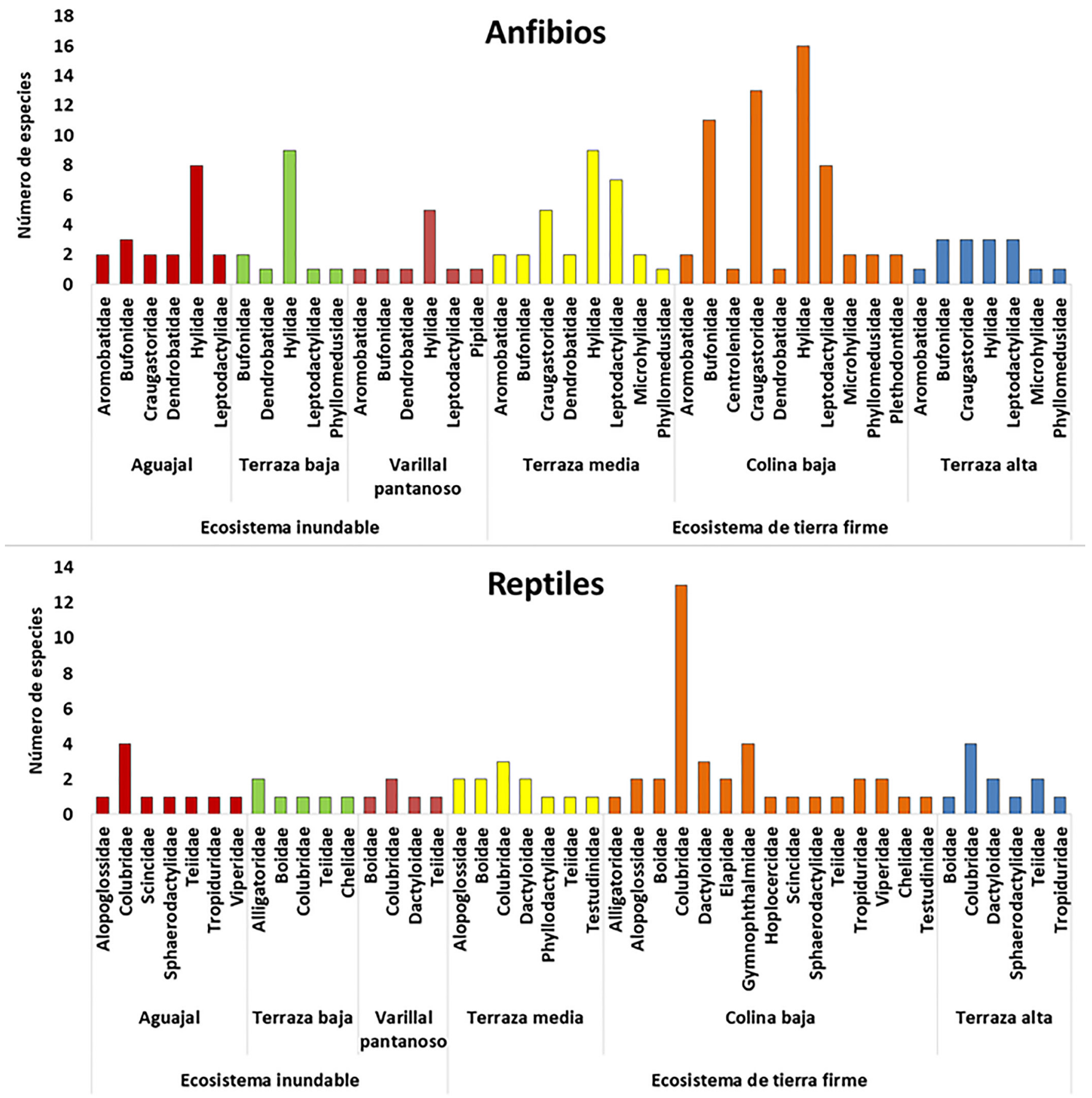

Figura 4: Riqueza de especies por familia y hábitats de anfibios y reptiles en el interfluvio Putumayo-Napo-Amazonas. 
Anfibios

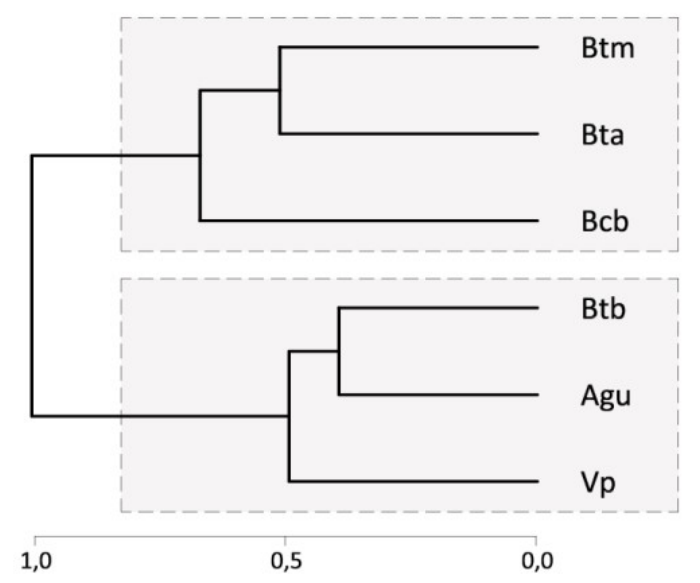

Reptiles

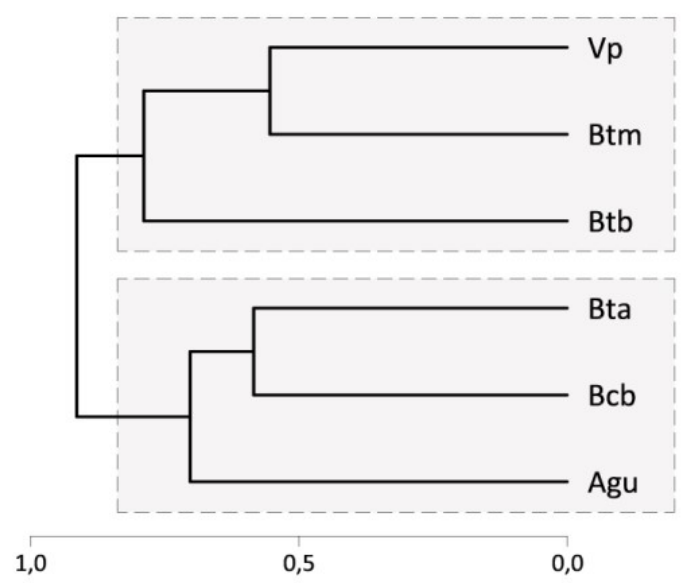

Figura 5: Similitud de hábitats basado en comunidades de anfibios y reptiles del interfluvio PutumayoNapo-Amazonas usando el índice de Sorensen. Los recuadros (gris) con líneas punteadas representan los grupos formados.

\section{SIMILITUD ENTRE HÁBITATS}

El análisis de similitud en las comunidades de anfibios por hábitats mostró dos grupos. Uno conformado por comunidades de: aguajal (Agu), terraza baja (Btb) y varillal pantanoso (Vp), y otro grupo conformado por: bosque de colina baja (Bcb), terraza alta (Bta) y terraza media (Btm) (Figura 5). El primer grupo está conformado por hábitats inundables, mientras el segundo grupo está conformado por los ecosistemas de tierra firme (Figura 5).

Las comunidades de reptiles también mostraron dos grupos. El primero conformado por: aguajal (Agu), bosque de colina baja $(\mathrm{Bcb}) \mathrm{y}$ terraza alta (Bta); y el segundo grupo conformado por: terraza baja (Btb), terraza media (Btm) y varillal pantanoso (Bp) (Figura 5). En todos los hábitats se notó una importante presencia de especies de la familia Colubridae sobre todo para el primer grupo.

\section{ESPECIE RESTRINGIDA AL INTERFLUVIO}

Ameerega bilinguis (Dendrobatidae) fue registrada en la cuenca baja del río Aguarico, cuenca baja del río Campuya, en la quebrada Bufeo de la cuenca baja del Putumayo, y al margen izquierdo del Amazonas, cerca de la desembocadura del río Napo a su margen izquierdo.

\section{ESPECIES AMENAZADAS}

El Decreto Supremo N004-2014-MINAGRI (MINAGRI, 2014) incluye en su lista a Podocnemis expansa en la categoría de especie en peligro (EN), mientras que Podocnemis unifilis y Podocnemis sextuberculata en la categoría de especie vulnerable (VU) (Tabla 4).

La IUCN (2020), incluye a Atelopus spumarius, Chelonoidis denticulata, Podocnemis unifilis y $P$. sextuberculata como especies vulnerables (VU) (Tabla 4). La lista CITES (2020) incluye a nueve especies anfibias y nueve de reptiles en el apéndice II de su lista, considerándolas como especies sensibles al comercio (Tabla 4).

Las tortugas dulceacuícolas componen la dieta alimenticia de muchas comunidades, estas son aprovechadas a nivel de carne y huevos las cuales son extraídas de las zonas de anidación. P. expansa tiene mayor tamaño y sus nidadas son 
Tabla 4. Especies de anfibios y reptiles del interfluvio Putumayo-Napo-Amazonas presentes en alguna categoría de conservación a nivel nacional o internacional.

\begin{tabular}{|c|c|c|c|c|c|}
\hline Clase & Familia & Especie & DS-004-2014 & IUCN & CITES \\
\hline \multirow{10}{*}{ Amphibia } & Aromobatidae & Allobates femoralis & & & II \\
\hline & Bufonidae & Atelopus spumarius & & VU & \\
\hline & \multirow{8}{*}{ Dendrobatidae } & Ameerega bilinguis & & & II \\
\hline & & Ameerega hahneli & & & II \\
\hline & & Ameerega parvula & & & II \\
\hline & & Ameerega trivittata & & & II \\
\hline & & Ranitomeya amazonica & & & ॥ \\
\hline & & Ranitomeya reticulata & & & II \\
\hline & & Ranitomeya variabilis & & & II \\
\hline & & Ranitomeya ventrimaculata & & & II \\
\hline \multirow{10}{*}{ Reptilia } & \multirow{3}{*}{ Alligatoridae } & Caiman crocodilus & & & $\|$ \\
\hline & & Melanosuchus niger & & & \\
\hline & & Paleosuchus trigonatus & & & II \\
\hline & \multirow{2}{*}{ Boidae } & Corallus batesii & & & ॥ \\
\hline & & Corallus hortulanus & & & II \\
\hline & Teiidae & Tupinambis teguixin & & & II \\
\hline & Testudinidae & Chelonoidis denticulata & & VU & II \\
\hline & \multirow{3}{*}{ Podocnemididae } & Podocnemis expansa & EN & & II \\
\hline & & Podocnemis sextuberculata & VU & VU & II \\
\hline & & Podocnemis unifilis & VU & VU & $\|$ \\
\hline \multicolumn{3}{|c|}{ Total } & 3 & 4 & 18 \\
\hline
\end{tabular}

más numerosas que $P$. unifilis y a diferencia de esta especie, prefiere playas más grandes para oviponer. Atelopus spumarius (Bufonidae) tiene un fuerte arraigo a los cuerpos de agua y son sensibles a la alteración del bosque.

\section{AMENAZAS Y RECOMENDACIONES PARA LA CONSERVACIÓN}

Una de las principales actividades que afectan a la riqueza de especies es la deforestación, ya sea con fines extractivos de madera o con fines agrícolas. Esta actividad favorece a la fragmentación de los bosques alterando el microclima de los diferentes hábitats de anfibios y reptiles. La cacería de caimanes y extracción de huevos de tortugas, especialmente de Podocnemis unifilis ("taricaya") es otra actividad que podría considerarse como amenaza para las poblaciones de estos grandes reptiles porque podría incrementarse en el tiempo. Estas actividades se incrementan en 
época de vaciante cuando las tortugas desovan en las playas y cuando los caimanes se restringen al cauce principal de los cuerpos de agua. La caza de caimanes también se da en tiempo de la recolección de alevinos de Osteoglosum bicirrhosum ("arahuana"), la cual es una de las actividades económicas principales en la cuenca baja del Putumayo. La carne de los caimanes y tortugas, además de los huevos de tortugas acuáticas, conforman parte importante de la dieta de los pobladores nativos del Putumayo.

\section{DISCUSIÓN}

El interfluvio alberga 140 especies de anfibios y 108 especies de reptiles, que equivale aproximadamente al 21,0\% y 21,3\% de las especies presentes en Perú (Frost, 2020; Uetz et al., 2020). Las 248 especies registradas en el presente trabajo es producto de más de 30 años de investigaciones, desde la publicación del clásico de Lescure \& Gasc, (1986) hasta los inventarios rápidos y expediciones que abarcaron aproximadamente un $80 \%$ del interfluvio Putumayo-Napo-Amazonas (Pitman et al., 2004; Alverson et al., 2008; Gilmore et al., 2010; Pitman et al., 2011; Knell, 2012; Pitman et al., 2013; Pitman et al., 2016; Pérez-Peña et al., 2019a; Pérez-Peña et al., 2019b; Pérez-Peña et al., en prensa), resultando en la protección de comunidades nativas y propuestas de nuevas áreas para conservación de la biodiversidad.

El encuadramiento de sinonimia y registro de especies nuevas, en anfibios, como por ejemplo del género Amazophrynella (Rojas et al., 2015; Rojas et al., 2018), Boana (Dubois, 2017), Callimedusa (Duellman et al., 2016), Dendropsophus (Wiens et al., 2010), Hypsiboas (Caminer \& Ron, 2014); Leptodactylus (de Sá et al., 2014), Pristimantis (Elmer \& Cannatella, 2008; Lehr et al., 2010; Moravec et al., 2010; Ortega-Andrade \& Venegas, 2014), Ranitomeya (Brown et al., 2011), Rhinella
(Pramuk, 2006), y en reptiles, Alopoglossus (Köhler et al., 2012), Copeoglossum, Mabuya y Varzea (Miralles et al., 2005, 2006, 2009; Hedges \& Conn, 2012), Loxopholis (Goicoechea et al., 2016), Thecadactylus (Bergmann \& Russell, 2007), Corallus batesii (Henderson et al., 2009), Erythrolamprus (Grazziotin et al., 2012), Bothrocophias (Carrasco et al., 2012), Chelonoidis (Olson \& David, 2014) jugaron un rol importante en el incremento de la riqueza de especies a lo largo de los años (Figura 2).

Por otro lado, se estima que la presencia de más especies de herpetozoos esté dispersa en todos los hábitats estudiados, respaldado por el estimador Chao 1. Los hábitats inundables por su maldrenaje, mantienen una humedad permanente durante periodos largos, favoreciendo la riqueza de especies de la familia Hylidae. Mientras que la riqueza de las especies terrestre-arbóreas de la familia Craugastoridae y Bufonidae, fueron altas en los ecosistemas de tierra firme. Las especies de la familia Colubridae tuvieron mayor riqueza en los ecosistemas de tierra firme y decreciendo en los ecosistemas inundables.

La familia Hylidae agrupa a especies arbóreas nocturnas, en su mayoría habitan desde bosques inundables hasta terrazas no inundables $y$ en diferentes estratos arbóreos, siendo más frecuentes en el suelo y el estrato medio (PalaciosRodríguez et al., 2018), sin embargo, por su fuerte dependencia a cuerpos de agua muchas especies podrían preferir las terrazas bajas inundables y aguajales.

La familia Craugastoridae, junto a la familia Hylidae, es una de las más ricas en número de especies (Frost, 2020), ocupando también estratos terrestres y arbóreos (Brito et al., 2017). Estas especies de acuerdo a su modo reproductivo (desarrollo directo) no están ligados a cuerpos de agua, sin embargo, hay especies que prefieren zonas próximas a quebradas o cuerpos de agua (Ramírez-Bautista, 2019), estas características les 
permite poblar de manera abundante bosques no inundables. Las especies de la familia Bufonidae son un grupo de anuros en su mayoría terrestres, aunque gran número de especies del género Rhinella duermen perchados a diferentes alturas en el estrato bajo del bosque (Duellman, 2005).

La familia Colubridae a pesar de ser la familia de reptiles más numerosa y de estar presente en diversos ecosistemas, es también un grupo menos estudiado (Rojas-Morales et al., 2018) a diferencia de los anfibios, esto podría deberse a que estas serpientes son más raras en los ecosistemas y para obtener buenos registros es necesario invertir mayor esfuerzo de muestreo.

Al parecer la distribución de Ameerega bilinguis (Dendrobatidae) se encuentra dentro de la ecorregión prioritaria de especies endémicas del neotrópico propuesta por Loyola et al. (2009), en la parte peruana. Actualmente, se cuenta con registros de esta especie en la cuenca baja del río Aguarico (Tapia-del Águila et al., 2019), cuenca baja del río Campuya (Venegas \& Gagliardi-Urrutia, 2013), en la quebrada Bufeo de la cuenca baja del Putumayo (Chávez \& Mueses-Cisneros, 2016) y en el bajo Amazonas, cerca de la desembocadura del río Napo a su margen izquierda (Tapia-del Águila et al., 2019).

De las 248 especies registradas en el interfluvio, 20 de ellas están incluidas en alguna categoría de conservación a nivel nacional o internacional (MINAGRI, 2014; IUCN, 2020; CITES, 2020). Asimismo, el estado de conservación de muchas de esas especies es deficiente o carecen de categorías, esto se debe a la descripción de nuevas especies cripticas y cambios taxonómicos, que, por falta de manejo de grandes cantidades de datos, dificultan la constante actualización de la lista de especies a nivel nacional y regional.

Estas especies incluidas bajo alguna categoría de amenaza se encuentran bajo presión antrópica. Entre los anfibios y reptiles, este último son más preferidos en la alimentación por su gran aporte proteico, entre ellos destacan los caimes y tortugas (Valencia-Aguilar et al., 2013). Es por ello, que el grado de afectación a los reptiles es directamente proporcional al tamaño, muchas especies tienen una estrecha relación con la economía de las comunidades nativas (Tapiadel Águila et al., 2019), siendo las especies más grandes las más utilizadas y por lo general, para consumo y comercio de carne y piel.

El interfluvio alberga una gran diversidad de anfibiosyreptilesquepuede ayudar enla economía del poblador amazónico, por ejemplo, mediante el manejo sostenible de carne de Caiman crocodilus y huevos de tortugas de Podocnemis unifilis. Estas especies son las más preferidas y tiene mayor precio de venta en el mercado, y pueden soportar un programa de aprovechamiento la cual ayudaría a mitigar la pobreza económica de los pobladores nativos y la conservación de este importante grupo de fauna silvestre.

\section{CONCLUSIONES}

Se registró 140 especies de anfibios y 108 especies de reptiles en el interfluvio Putumayo-NapoAmazonas. Las familias más representativas en anfibios fueron Hylidae y Craugastoridae con 38 y 32 especies, respectivamente. Mientras, en reptiles la familia Colubridae tuvo la mayor riqueza con 39 especies. Los bosques de colina tuvieron el mayor número de especies de anfibios y reptiles, mientras que el bosque de terraza baja y varillal pantanoso fueron los que tuvieron menor riqueza de especies. Las comunidades de anfibios se diferencian muy bien de acuerdo al bosque inundable y no inundable, los reptiles también siguen el mismo patrón. La especie de la familia Dendrobatidae, Ameerega bilinguis, es la especie que está restringida a este interfluvio. Del total de las especies registradas, tres especies se encuentran incluidas en la categoría de protegidas 
por la legislación nacional peruana, de las cuales dos especies se encuentran en la categoría de vulnerable y una especie en la categoría en peligro. La deforestación con fines extractivos y agrícolas, y además de la cacería de reptiles grandes, son las principales amenazas en el interfluvio. Para ello es necesario tener planes de manejo que ayuden a garantizar la conservación y comprensión de los servicios ecosistémicos de estas especies.

\section{REFRENCIAS BIBLIOGRÁFICAS}

Alverson, W.S.; Vriesendorp, C.; del Campo, A.; Moskovits, D.K.; Stotz, D.F.; García, M.; Borbor L.A. 2008. Ecuador, Perú: Cuyabeno - Güeppí. Rapid Biological and Social Inventories, 20. The Field Museum, Chicago. 376pp.

Aguilar, C.; Ramírez, C.; Rivera, D.; Siu-Ting, K.; Suarez, J.; Torres, C. 2010. Anfibios andinos del Perú fuera de Áreas Naturales Protegidas: amenazas y estado de conservación. Revista Peruana de Biología, 17(1): 5-28. DOI: https:// doi.org/10.15381/rpb.v17i1.46

Bergmann, P.J.; Russell, A.P. 2007. Systematics and biogeography of the widespread neotropical gekkonid genus Thecadactylus (Squamata), with the description of a new cryptic species. Zoological Journal of the Linnean Society, 149(3): 339-370. DOI: https://doi. org/10.1111/j.1096-3642.2007.00251.x

Brito, M.J.; Almendáriz, C.A.; Batallas, R.D.; Ron, S.R. 2017. Nueva especie de rana bromelícola del género Pristimantis (Amphibia: Craugastoridae), meseta de la Cordillera del Cóndor, Ecuador. Papéis Avulsos De Zoologia, 57(15): 177-195. https://doi. org/10.11606/0031-1049.2017.57.15

Brown, J.L.; Twomey, E.; Amézquita, A.; De Souza, M.B; Caldwell, J.P.; Lötters, S.; von May, R.; MeloSampaio, P.R.; Mejía-Vargas, D.; Pérez-Peña, P.; Pepper, M.; Poelman, E.H.; Sanchez-Rodriguez,
M.; Summers, K. 2011. A taxonomic revision of the neotropical poison frog genus Ranitomeya (Amphibia: Dendrobatidae). Zootaxa, 3083(1): 1-120. DOI: https://doi.org/10.11646/ zootaxa.3083.1.1

Caminer, M.A.; Ron, S.R. 2014. Systematics of treefrogs of the Hypsiboas calcaratus and Hypsiboas fasciatus species complex (Anura, Hylidae) with the description of four new species. ZooKeys, 370: 1-68. DOI: https://doi. org/10.3897/zookeys.370.6291

Carrasco, P.A.; Mattoni, C.I.; Leynaud, G.C.; Scrocchi, G.J. 2012. Morphology, phylogeny and taxonomy of South American bothropoid pitvipers (Serpentes, Viperidae). Zoologica Scripta, 41(2): 109-124. DOI: https://doi. org/10.1111/j.1463-6409.2011.00511.x

Chávez, G.; Mueses-Cisneros, J.J. 2016. Anfibios y reptiles. In: Pitman, N.; Bravo, A.; Claramunt, S.; Vriesendorp, C.; Alvira, D.; Ravikumar, A.; del Campo, A.; Stotz, D.F.; Wachter, T.; Heilpern, S.; Rodríguez, B.; Sáenz, A.R; Smith, R.C. (Eds). Perú: Medio Putumayo - Algodón. Rapid Biological and Social Inventories, 28. The Field Museum, Chicago, USA. p. 107-113.

CITES (Convención sobre el Comercio Internacional de Especies Amenazadas de Fauna y Flora Silvestres). 2020. Apéndice I, II y III. (https://www.cites.org/esp). Acceso: 05/09/2020.

Climate-Data. 2018. Climate: El Estrecho. (https://es.climate-data.org/america-del-sur/ peru/loreto/el-estrecho-45827/). Acceso: 22/07/2019.

Crump, M.L.; Scott, N.J. 2001. Relevamientos por encuentros visuales. In: Heyer, W.R.; Donelly, M.A.; Mc Diarmid, R.W.; Hayek, L.C.; Foster, M. (Eds). Medición y monitoreo de la diversidad biológica. Métodos estandarizados para anfibios. p. 80-87.

da Cunha Bitar; Y.O.; Juen, L.; Pinheiro, L.C.; SantosCosta, M.C.D. 2015. Anuran beta diversity in a 
mosaic anthropogenic landscape in transitional Amazon. Journal of Herpetology, 49(1): 75-82. DOI: https://doi.org/10.1670/13-041

de Sá, R.O.; Grant, T.; Camargo, A.; Heyer, W.R.; Ponssa, M.L.; Stanley, E. 2014. Systematics of the neotropical genus Leptodactylus Fitzinger, 1826 (Anura: Leptodactylidae): phylogeny, the relevance of non-molecular evidence, and species accounts. South American Journal of Herpetology, 9(1): 1-128. DOI: https://doi. org/10.2994/SAJH-D-13-00022.1

Dinerstein, E.; Olson, D.; Joshi, A.; Vynne, C.; Burgess, N.D.; Wikramanayake, E.; Hahn, N.; Palminteri, S.; Hedao, P.; Noss, R.; Hansen, M.; Locke, H.; Ellis, E.C.; Jones, B.; Barber, C.V.; Hayes, R.; Kormos, C.; Martin, V.; Crist, E.;

[...]; Saleem, M. 2017. An ecoregion-based approach to protecting half the terrestrial realm. BioScience, 67(6): 534-545. DOI: https://doi.org/10.1093/biosci/bix014

Doan, T.M. 2003. Which methods are most effective for surveying rain forest herpetofauna?. Journal of Herpetology, 37(1): 72-81. DOI: https://doi.org/10.1670/00221511(2003)037[0072:WMAMEF]2.0.CO;2

Dubois, A. 2017. The nomenclatural status of Hysaplesia, Hylaplesia, Dendrobates and related nomina (Amphibia, Anura), with general comments on zoological nomenclature and its governance, as well as on taxonomic databases and websites. Bionomina, 11(1): 1-48. DOI: https://doi.org/10.11646/bionomina.11.1.1

Duellman, W.E. 2005. Cusco amazonico: the lives of amphibians and reptiles in an amazonian rainforest. Cornell University Press, Ithaca (New York). 433pp.

Duellman, W.E.; Marion, A.B.; Hedges, B. 2016. Phylogenetics, classification and biogeography of the treefrogs (Amphibia: Anura: Arboranae). Zootaxa, 4104(1): 1-109. DOI: https://doi. org/10.11646/zootaxa.4104.1.1
Elmer, K.R.; Cannatella, D.C. 2008. Three new species ofleaflitter frogs from the upper Amazon forests: cryptic diversity within Pristimantis "ockendeni" (Anura: Strabomantidae) in Ecuador.Zootaxa, 1784(1): 11-38. DOI: https:// doi.org/10.11646/zootaxa.1784.1.2

MINAGRI. 2014. Decreto Supremo $\mathrm{N}^{\circ}$ 004-2014-MINAGRI: Aprueba la actualización de la lista de clasificación y categorización de las especies amenazadas de fauna silvestre legalmente protegidas. Diario Oficial El Peruano. 08 de abril del 2014.

Frost, D.R. 2020. Amphibian species of the world: an online reference. American Museum of Natural History. (http://research.amnh.org/ herpetology/amphibia/index.html). Acceso: 05/09/2020.

Gallmetzer, N.; Schulze, C.H. 2015. Impact of oil palm agriculture on understory amphibians and reptiles: a mesoamerican perspective. Global Ecologyand Conservation, 4: 95-109. DOI: https://doi.org/10.1016/j.gecco.2015.05.008

Gilmore, M.P.; Vriesendorp, C.; Alverson, W.S.; del Campo, A.; von May, R.; López, C.; Ríos, S. 2010. Perú: Maijuna. Rapid Biological and Social Inventories, 22. The Field Museum, Chicago. 328pp.

Goicoechea, N.; Frost, D.R.; De la Riva, I.; Pellegrino, K.C.M.; Sites, J.; Rodrigues, M.T.; Padial, J.M. 2016. Molecular systematics of teioid lizards (Teioidea/Gymnophthalmoidea: Squamata) based on the analysis of 48 loci under treealignment and similarity-alignment. Cladistics, 32(6): 624-671. DOI: https://doi.org/10.1111/ cla. 12150

Grazziotin, F.G.; Zaher, H.; Murphy, R.W.; Scrocchi, G.; Benavides, M.A.; Zhang, Y.P.; Bonatto, S.L. 2012. Molecular phylogeny of the New World Dipsadidae (Serpentes: Colubroidea): a reappraisal. Cladistics, 28(5): 437-459. DOI: $\quad$ https://doi.org/10.1111/j.10960031.2012.00393.x 
Hedges, S.B.; Conn, C.E. 2012. A new skink fauna from Caribbean islands (Squamata, Mabuyidae, Mabuyinae). Zootaxa, 3288(1): 1-244. DOI: https://doi.org/10.11646/zootaxa.3288.1.1

Henderson, R.W.; Passos, P.; Feitosa, D. 2009. Geographic variation in the emerald treeboa, Corallus caninus (Squamata: Boidae). Copeia, 2009(3): 572-582. DOI: https://doi. org/10.1643/CH-08-190

IUCN (The International Union for Conservation of Nature). 2020. The IUCN red list of threatened species, version 2020-2. (http:// www.iucnredlist.org). Acceso: 04/09/2020.

Knell, G. 2012. Diagnóstico del potencial turístico del sector Lagartococha-Zona Reservada de Guëppi. SERNANP, Lima. 67pp.

Köhler, G.; Hans-Helmut, D.; Veselý, M. 2012. A contribution to theknowledgeofthelizardgenus Alopoglossus (Squamata: Gymnophthalmidae). Herpetological Monographs, 26(1): 17388. DOI: https://doi.org/10.1655/ HERPMONOGRAPHS-D-10-00011.1

Lehr, E.; Moravec, J.; Gagliardi-Urrutia, L.A.G. 2010. A new species of Pristimantis (Anura: Strabomantidae) from the Amazonian lowlands of northern Peru. Salamandra, 46(4): 197-203.

Lescure, J.; Gasc, J.P. 1986. Partage de l'espace forestier par les amphibiens et les reptiles en Amazonie du nord-ouest. Caldasia, 15(71-75): 707-723.

López-Rojas, J.J.; Cisneros-Heredia, D.F. 2012. Synapturanus rabus Pyburn, 1977 in Peru (Amphibia: Anura: Microhylidae): filling gap. Check List, 8(2): 274-275. DOI: https:// doi.org/10.15560/8.2.274

Loyola, R.D.; Kubota, U.; da Fonseca, G.A.B.; Lewinsohn, T.M. 2019. Key Neotropical ecoregions for conservation of terrestrial vertebrates. Biodiversity and Conservation, 18(8): 2017-2031. DOI: https://doi. org/10.1007/s10531-008-9570-6
Maretti, C.C.; Riveros, S.J.C.; Hofstede, R.; Oliveira, D.; Charity, S.; Granizo, T.; Alvarez, C.; Valdujo, P.; Thompson, C. 2014. State of the Amazon: ecological representation in protected areas and indigenous territories. WWF, Brasília. 82pp. MINAM (Ministerio del Ambiente). 2015. Mapa nacional de cobertura vegetal: memoria descriptiva. Ministerio del Ambiente, Lima. 100pp.

Miralles, A.; Rivas-Fuenmayor, G.; Barrio-Amorós, C.L. 2005. Taxonomy of the genus Mabuya (Reptilia, Squamata, Scincidae) in Venezuela. Zoosystema, 27(4): 825-37.

Miralles, A.; Barrio-Amorós, C.L.; Rivas, G.; Chaparro-Auza, J.C. 2006. Speciation in the «Várzéa» flooded forest: a new Mabuya (Squamata, Scincidae) from Western Amazonia. Zootaxa, 1188(1): 1-22. DOI: https://doi. org/10.11646/zootaxa.1188.1.1

Miralles, A.; Chaparro, J.C.; Harvey, M.B. 2009. Three rare and enigmatic South American skinks. Zootaxa, 2012(1): 47-68. DOI: https:// doi.org/10.11646/zootaxa.2012.1.3

Moravec, J.; Lehr, E.; Pérez-Peña, P.E.; López, J.J.; Gagliardi-Urrutia, L.A.G.; Arista-Tuanama, I. 2010. A new green, arboreal species of Pristimantis (Anura: Strabomantidae) from Amazonian Peru. Vertebrate Zoology, 60(3): 225-232.

Olson, S.L.; David, N. 2014. The gender of the tortoise genus Chelonoidis Fitzinger, 1835 (Testudines: Testudinidae). Proceedings of the Biological Society of Washington, 126(4): 393-394. DOI: https://doi.org/10.2988/0006324X-126.4.393

Ortega-Andrade, H.M.; Venegas, P.J. 2014. A new synonym for Pristimantis luscombei (Duellman and Mendelson 1995) and the description of a new species of Pristimantis from de upper Amazon basin (Amphibia: Craugastoridae). Zootaxa, 3895(1): 31-57. DOI: https://doi. org/10.11646/zootaxa.3895.1.2 
Ossa, P.A.; Giraldo, J.M.; López, G.A.; Dias, L.G.; Rivera, F.A. 2012. Colecciones biológicas: una alternativa para los estudios de diversidad genética. Boletín Científico. Centro de Museos Museo de Historia Natural, 16(1): 143-155.

OTCA (Organización del Tratado de Cooperación Amazónica). 2018. La importancia de las colecciones biológicas para la conservación de la biodiversidad. (http://www.otca-oficial. info/news/details/681). Acceso: 21/09/2020.

Palacios-Rodríguez, L.; Rengifo-Mosquera, J.; Roa, M.; Palacios, Y. 2018. Riqueza y distribución de Hílidos (Hylidae: Anura) en zona de bosques pluvial tropical en el departamento del Chocó, Colombia. Revista Colombiana De Ciencia Animal, 10(2): 154-166. DOI: https://doi. org/10.24188/recia.v10.n2.2018.650

Pérez-Peña, P.E.; Ramos-Rodríguez, M.C.; Díaz, J.; Zárate, R.; Mejía, K. 2019a. Biodiversidad en la cuenca alta del Putumayo, Perú. Instituto de Investigaciones de la Amazonía Peruana, Iquitos. 148pp.

Pérez-Peña, P.E.; Ramos-Rodríguez, M.C.; Díaz, J.; Zárate, R.; Mejía, K. 2019b. Biodiversidad en las cuencas del Napo y Curaray, Perú. Instituto de Investigaciones de la Amazonía Peruana; Centro para el Desarrollo del Indígena Amazónico, Iquitos. 203pp.

Pérez-Peña, P.E; Medina, I.P.; Pizarro, J.S. 2019c. Anfibios y reptiles en bosque inundable y tierra firme. In: Pérez-Peña, P.E.; Ramos-Rodríguez, M.C.; Díaz, J.; Zárate, R.; Mejía, K. (Eds). Biodiversidad en la cuenca alta del Putumayo, Perú. p. 81-107.

Pérez-Peña, P.E.; Ramos-Rodríguez, M.C.; Mejía, K. En Prensa. Biodiversidad en la cuenca baja del Putumayo, Perú. Instituto de Investigaciones de la Amazonía Peruana, Iquitos. s.p.

Pitman, N.; Smith, R.C.; Vriesendorp, C.; Moskovits, D.; Piana, R.; Knell G.; Wachter T. 2004. Perú: Ampiyacu, Apayacu, Yaguas, Medio Putumayo.
Rapid Biological and Social Inventories, 12. The Field Museum, Chicago, Illinois. 273pp.

Pitman, N.; Vriesendorp, C.; Moskovits, D.K.; von May, R., Alvira, D.; Wachter, T.; Stotz, D.F.; del Campo, A. 2011. Perú: Yaguas-Cotuhé. Rapid Biological and Social Inventories, 23. The Field Museum, Chicago. 378pp.

Pitman, N.; Ruelas, E.; Vriesendorp, C.; Stotz, D.F.; Watchter, T.; del Campo, A.; Alvira, D.; Rodríguez, B.; Smith, R.C.; Sáenz, A.R.; Soria, P. 2013. Perú: Ere-Campuya-Algodón. Rapid Biological and Social Inventories, 25. The Field Museum, Chicago. 404pp.

Pitman, N.; Bravo, A.; Claramunt, S.; Vriesendorp, C.; Alvira, D.; Ravikumar, A.; del Campo, A.; Stotz, D.F.; Wachter, T.; Heilpern, S.; Rodríguez, B.; Sáenz, A.R.; Smith, R.C. 2016. Perú: Medio Putumayo - Algodón. Rapid Biological and Social Inventories, 28. The Field Museum, Chicago. 522pp.

Pramuk, J.B. 2006. Phylogeny of south american Bufo (Anura: Bufonidae) inferred from combined evidence. Zoological Journal of the Linnean Society 146(3): 407-452. DOI: https:// doi.org/10.1111/j.1096-3642.2006.00212.x

Ramírez-Bautista, N.R. 2019. Descripción del uso de sustrato de Pristimantis savagei (Pyburn \& Lynch, 1981) (Anura: Craugastoridae) del bosque de los Guayupes (Acacías, MetaColombia). Tesis de pre-grado. Universidad de los Llanos. Facultad de Ciencias Básicas e Ingeniería, Villavicencio, Colombia. 45pp.

Rodríguez, L.O.; Knell, G. 2004. Anfibios y reptiles. In: Pitman, N.; Smith, R.C.; Vriesendorp, C.; Moskovits, D.; Piana, R.; Knell, G.; Wachter, T. (Eds). Perú: Ampiyacu, Apayacu, Yaguas, Medio Putumayo. Rapid Biological and Social Inventories, 12. p. 67-71.

Rojas, R.; Carvalho, V.T.; Ávila, R.W.; Farias, I.P.; Gordo, M.; Hrbek, T. 2015. Two new species of Amazophrynella (Amphibia: Anura: Bufonidae) 
from Loreto, Peru. Zootaxa, 3946(1): 79-103. DOI: https://doi.org/10.11646/zootaxa. 3946.1.3

Rojas, R.R.; Fouquet, A.; Ron, S.R.; Hernández-Ruz, E.J.; Melo-Sampaio, P.R.; Chaparro, J.C.; Vogt, R.C.; Carvalho, V.T.d.; Pinheiro, L.C.; Avila, R.W.; Farias, I.P.; Gordo, M.; Hrbek, T. 2018. A PanAmazonian species delimitation: high species diversity within the genus Amazophrynella (Anura: Bufonidae). PeerJ, 6: e4941. DOI: https://doi.org/10.7717/peerj.4941

Rojas-Morales, J.A.; Marín-Martínez, M.; ZuluagaIsaza, J.C. 2018. Aspectos taxonómicos y ecogeográficos de algunas serpientes (Reptilia: Colubridae) del área de influencia de la Central Hidroeléctrica Miel I, Caldas, Colombia. Biota Colombiana, 19(2): 73-9. DOI: http://dx.doi. org/10.21068/c2018.v19n02a07

RStudio Team. 2019. RStudio: integrated development for R. RStudio, (https://www.rstudio. com). Acceso: 19/12/2019.

Tapia-del Águila, C.; Arévalo-Piña, I.; Pérez-Peña, P.; Sánchez, M. 2019. Anfibios y Reptiles. In: Pérez-Peña, P.E.; Ramos-Rodríguez, M.C.; Díaz, J.; Zárate, R.; Mejía, K. (Eds). Biodiversidad en las cuencas del Napo y Curaray, Perú. Instituto de Investigaciones de la Amazonía Peruana; Centro para el Desarrollo del Indígena Amazónico. p. 71-97.

Tapia-del Águila, C.J.; Pérez-Peña, P.E. En prensa. Anfibios y reptiles de terrazas no inundables. In: Pérez-Peña, P.E.; Ramos-Rodríguez, M.C.; Mejía, K. (Eds). Biodiversidad en la cuenca baja del Putumayo, Perú. s.p.

Uetz, P.; Freed, P.; Hošek, J.J. 2020. The reptile database. (http://www.reptile-database.org). Acceso: 05/09/2020.

Valencia-Aguilar, A.; Cortés-Gómez, A.M.; RuizAgudelo; C.A. 2013. Ecosystem services provided by amphibians and reptiles in neotropical ecosystems. International Journal of Biodiversity Science, Ecosystem Services \& Management, 9(3): 257-272. DOI: https://doi. org/10.1080/21513732.2013.821168

Venegas, P.J.; Gagliardi-Urrutia, G. 2013. Anfibios y reptiles. In: Pitman, N.; Ruelas, E.; Vriesendorp, C.; Stotz, D.F.; Watchter, T.; del Campo, A.; Alvira, D.; Rodríguez, B.; Smith, R.C.; Sáenz, A.R.; Soria, P. (Eds). Perú: Ere-Campuya-Algodón. Rapid Biological and Social Inventories, 25. p. 119131.

von May, R.; Venegas, P.J. 2010. In: Gilmore, M.P.; Vriesendorp, C.; Alverson, W.S.; del Campo, A.; von May, R.; López, C.; Ríos, S. (Eds). Perú: Maijuna. Rapid Biological and Social Inventories, 22. p. 74-81.

von May, R.; Mueses-Cisneros, J.J. 2011. Anfibios y reptiles. In: Pitman, N.; Vriesendorp, C.; Moskovits, D.K.; von May, R.; Alvira, D.; Wachter, T.; Stotz, D.F.; del Campo, A. (Eds). Perú: YaguasCotuhé. Rapid Biological and Social Inventories, 23. p. 108-116.

Wiens, J.J.; Kuczynski, C.A.; Hua, X.; Moen, D.S. 2010. An expanded phylogeny of treefrogs (Hylidae) based on nuclear and mitochondrial sequence data. Molecular Phylogenetics and Evolution, 55(3): 871-882. DOI: https://doi. org/10.1016/j.ympev.2010.03.013

Yáñez-Muñoz, M.; Venegas, P.J. 2008. In: Alverson, W.S.; Vriesendorp, C.; del Campo, A.; Moskovits, D.K.; Stotz, D.F.; García, M.; Borbor, L.A. (Eds). Ecuador, Perú: Cuyabeno - Güeppí. Rapid Biological and Social Inventories, 20. p. 90-96.

Zarate-Gómez, R.; Cohello-Huaymacari, G.; Palacios, J.J.; Escobedo-Torres, R.; Mejía, K.; Calvache-Torres, S.; Vasquez-Mananita, V. 2019. Vegetación y flora. In: Pérez-Peña, P.E.; RamosRodríguez, M.C.; Díaz-Alván, J.; Zarate-Gómez, R.; Mejía-Carhuanca, K. (Eds). Biodiversidad en la cuenca alta del Putumayo, Perú. p. 19-60.

Recibido: 26 de setiembre de 2020 Aceptado para publicación: 16 de diciembre de 2020

Esta obra está bajo una Licencia Creative Commons Atribución-NoComercial-SinDerivar 4.0 Internacional. 